Exploring Photoinduced Intermolecular Electron transfer with

Local Control Theory in Trajectory-based Nonadiabatic Dynamics

\section{Molecular Dynamics Simulations}

\section{A. Gamiz-Hernandez ${ }^{1}$, E. Vauthey ${ }^{2}$, M. Meuwly ${ }^{1}$}

${ }^{1}$ University of Basel/Department of Chemistry, Klingelbergstr. 80, CH-4055 Basel, Switzerland

${ }^{2}$ University of Geneva/Department of Physical Chemistry, 30 quai ErnestAnsermet, CH-1211 Geneva 4, Switzerland

Understanding the dynamics of photoinduced chemical processes involving electron transfer (ET) is a crucial step for the design of new technologies such as efficient solar energy conversion devices. Photoinduced ET processes have been investigated for some time [1], nevertheless there are still several questions regarding intermolecular ET reactions such as the occurrence of long distance reactions and the Charge Separation (CS) products.

Many experiments have corroborated Marcus theory [2] for ET, however, most of these models are simplifications as they consider the reactants as spheres and quantities such as the reorganization energy are computed using continuum models for the solvent. Molecular Dynamics (MD) simulations of photoinduced reactions have the potential to provide a better insight to explore for example, at what distance CS takes place, what is the nature of the primary ion pair product, computation of more accurate reorganization energies based on an explicit solvent models and how intermolecular interactions influence the orientations of the reactants.

This research will present some results from MD Simulations of bimolecular photoinduced ET reactions [1], in particular the PeryleneDimethylaniline system. We investigate how the solvent adopts different configurations as well as possible pathways for CS. These conformations are used for quantum-chemical calculations to generate absorption/emission spectra, helping to explain experimental results.

[1] E. Vauthey, J. Photochem. Photobiol. A, 2006, 179, 1.

[2] R. A. Marcus and N. Sutin, Biochim. Biophys. Acta, 1985, 811, 265.

\section{B. F. E. Curchod $^{1}$, T. J. Penfold ${ }^{1,2,3}$, U. Rothlisberger ${ }^{1}$, and I. Tavernelli ${ }^{1}$}

${ }^{1}$ Laboratory of Computational Chemistry and Biochemistry, Ecole Polytechnique Fédérale de Lausanne, CH-1015 Lausanne, Switzerland ${ }^{2}$ Laboratoire de Spectroscopie Ultrarapide, Ecole Polytechnique Fédérale de Lausanne, CH-1015 Lausanne, Switzerland

${ }^{3}$ SwissFEL, Paul Scherrer Inst., CH-5232 Villigen, Switzerland.

In the last years, linear-response time-dependent density functional theory (LR-TDDFT) has become widely used for the calculation of vertical electronic excitation energies of medium to large size molecular systems in gas and condensed phases. In addition, excited state LR-TDDFT forces and nonadiabatic couplings have also been implemented in different software packages, which enables LR-TDDFT based ab initio molecular dynamics approaches for the study of photochemical reactions.

Here, we present a development of a LR-TDDFT trajectory surface hopping scheme that couples nuclear and electronic degrees of freedom with an external electromagnetic field $[1,2]$. This further allows the on-the-fly calculation of an electric pulse, which controls the population transfer between electronic states using local control theory. This method is applied to the molecule lithium fluoride and the results are compared with nuclear quantum dynamics using the MCTDH method. In addition, we will present an example of how a controlled pulse can trigger a photochemical reaction like proton transfer in 4-hydroxyacridine.

[1] I. Tavernelli, B. F. E. Curchod, and U. Rothlisberger, Phys. Rev. A 2010, 81,052508 .

[2] B. F. E. Curchod, T. J. Penfold, U. Rothlisberger, and I. Tavernelli, Phys. Rev. A 2011, 84, 042507.
Computational Chemistry, Talk

Effect of Protein Environment on Excitation Energy of Retinal

$\underline{\text { Xiuwen Zhou }}^{1}$, Tomasz A. Wesolowski ${ }^{2}$

${ }^{1}$ University of Geneva, Department of Physical Chemistry, Quai ErnestAnsermet 30, CH-1211 Geneva, Switzerland

Retinal is a chromophore of key importance in the photochemical process responsible for animal vision, which begins with cis-trans photoisomerization (see Figure 1) of retinal bound to opsin (a protein) [1] The absorbtion spectrum of the chromophore is affected by the protein to which it is bound. The long range objective of this project is the development of an accurate modeling technique to predict the effect of protein mutations on the absorption spectra of retinal. Such technique could be used to help designing protein mutations leading to desired spectral properties of the chromophore. The applied computational method belongs to the category of multi-level simulations and is based on Frozen-Density Embedding Theory (FDET) [2-4]. The results of the first stage of this project will be reported concerning: a) development of an efficient method to generate the frozen density for FDET calculations developed based on comparison between benchmark supermolacular ab-initio results [5] obtained for small models of the environment, and b) modeling the effect of point mutations in proteins on the absorption spectra.

Figure 1. Photoisomerization of retinal

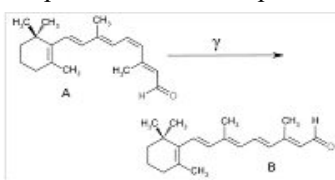

[1] R. Hubbard and G. Wald, J. Gen. Physiol., 1952-53, 36, 269.

[2] T.A. Wesolowski and A. Warshel, J. Phys. Chem., 1993, 97, 8050.

[3] T.A. Wesolowski, Computational Chemistry: Reviews of Current

Trends, J. Leszczynski, Ed. World Scientific, 2006, 10, 1.

[4] T.A. Wesolowski, Phys. Rev. A, 2008, 77, 012504.

[5] V.R.I. Kaila, R. Send, D. Sundholm, J. Phys. Chem. B, 2012, 116, 2249
Computational Chemistry, Talk

\section{Spectral Tuning in Rhodopsin Early Photointermediates}

\section{Pablo Campomanes, Ursula Röthlisberger}

Ecole Polytechnique Fédérale Lausanne, CH-1015, Lausanne, Switzerland

The visual pigment rhodopsin is a highly specialized member of the GPCR family found in vertebrate rod cells. Rhodopsin is able to capture and convert light into a chemical signal, in what constitutes the first step of the vision. The photoinduced isomerization of its natural ligand (11-cis retinal) inside the binding pocket initiates a cascade of conformational changes that leads ultimately to receptor activation and subsequent downstream signaling. Several spectroscopically distinguishable intermediates involved in the early steps of this photoactivation mechanism have been detected, and the structures of some of them have been determined. However, the complete knowledge of the factors that selectively modulate retinal absorption during the initial events of the visual cascade is far from being achieved and, therefore, a direct relation between the structural changes caused by the relaxation of the photoexcited chromophore upon isomerization and the corresponding optical shifts observed for the intermediates involved in the very early steps of the rhodopsin photocycle has not been established yet.

In this contribution, I will show how a combined strategy based on the computation of absorption energies, using the ZINDO/S semi-empirical method, for a statistically relevant number of thermally sampled configurations extracted from QM/MM trajectories can be used to establish a one-to-one correspondence between the structures of the different intermediates involved in the very early steps of the rhodopsin photocycle and their optical spectra. The analysis of the results clarify the role played by some of the residues located in the protein-binding pocket as well as the importance of the structural features and conformation of the chromophore on the spectral shifts observed between the intermediates. They also permit the identification of the structural parameters that better describe the different transitions leading from dark- to lumi-rhodopsin, therefore allowing the characterization of the distinct states. 
Computational Chemistry, Talk

Unravelling the electronic structure of molecules with density-only based detectors

$\underline{\text { Piotr de Silva }}^{1,2}$, Jacek Korchowiec ${ }^{1}$, Tomasz A. Wesolowski ${ }^{2}$

${ }^{1} \mathrm{~K}$. Gumiński Department of Theoretical Chemistry, Faculty of Chemistry, Jagiellonian University, R. Ingardena 3, 30-060 Kraków, Poland

${ }^{2}$ Université de Genève, Département de Chimie Physique 30, quai Ernest-Ansermet, CH-1211 Genève 4, Switzerland

The Electron Localization Function (ELF) [1-3] has proved to be an exceptionally useful concept in the elucidation of bonding patterns in molecules and solids. The major drawbacks of ELF are its orbital dependence and the use of the homogeneous electron gas a reference. In this contribution, we propose an entirely new paradigm for the clarification of the electronic structure in terms of such intuitive concepts like bonds, core electrons and lone pairs. We introduce detectors which are calculated only from the electron density and its first and second derivatives. This feature makes them directly applicable at any level of theory as well as to experimental densities.
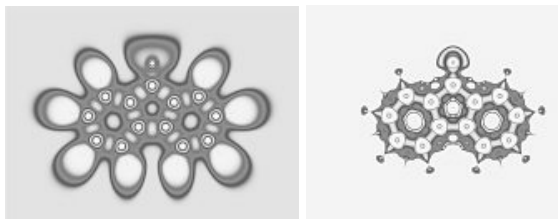

Figure 1: ELF (left) and one of the proposed detectors (right) for the fluorenone molecule.

[1] Becke, A. D. and Edgecombe, K. E., J. Chem. Phys. 1990, 92, 5397.

[2] Silvi, B. and Savin, A. , Nature 1994, 371, 683.

[3] Savin, A.; Nesper, R.; Wengert, S.; Fassler, T. F., Angew. Chem. Int. Ed. Engl. 1997, 36, 1809.

New advances in adiabatic reactive molecular dynamics simulations

Tibor Nagy, Juvenal Yosa, Markus Meuwly*

University of Basel, Klingelbergstrasse 80, 4056 Basel

Adiabatic reactive molecular dynamics (ARMD) simulation is a surfacecrossing algorithm [1-4] that allows one to follow bond-breaking and bondforming processes in classical molecular dynamics simulations.

In ARMD the dynamics of the system is propagated on the $V_{0}(x)=\min \left[V_{\mathrm{R}}(x)+\delta, V_{\mathrm{P}}(x)\right]$ surface, where $V_{\mathrm{R}}$ and $V_{\mathrm{P}}$ are the potential energy surfaces of reactants and products, respectively, and $\delta$ is used to adjust the asymptotics of the two surfaces. During dynamics when the energy of the two surfaces become equal, the system is re-simulated from a while earlier by gradually switching in time from $V_{\mathrm{R}}+\delta$ to $V_{\mathrm{P}}$ thus surface crossing can take place.

Although the total energy can be approximately conserved over the short switching times [1], the algorithm can be improved. Furthermore depending on the speed of approach to the barrier different barrier region is felt by the atoms and even spurious transient maxima can appear. These artifacts may alter the dynamics during crossing significantly and lead to different final-state specific reaction probabilities. An alternative geometry-based switching function was proposed, which cures all these problems and eliminates the need for repeated simulation.

The new algorithm is applied to the unimolecular decomposition of sulphuric acid after overtone excitation of the $\mathrm{OH}$ vibration $[3,5]$, and to the charge-transfer reaction between $\mathrm{N}_{2}$ and $\mathrm{N}_{2}{ }^{+}$at low temperatures. The differences compared to the original approach are discussed.

[1] J. Danielsson, M. Meuwly, J. Chem. Theory. Comput. 2008, 4, 1083.

[2] S. Mishra, M. Meuwly. J. Am. Chem. Soc. 2010, 132, 2968.

[3] J. Yosa, M. Meuwly. J. Phys. Chem. B 2011, 115, 14350.

[4] S. Lutz, M. Meuwly. ChemPhysChem 2012, 13, 305.

[5] V. Vaida, D.J. Donaldson, H.G. Kjaergaard, P.E. Hintze Science 2003, 299, 1566
Computational Chemistry, Talk

Exploring the Limits of Modern Density Functional Approximations for Interaction Energies

\section{Stephan N. Steinmann and Clemence Corminboeuf}

Laboratory for Computational Molecular Design, Institute of Chemical Sciences and Engineering, Ecole Polytechnique Fédérale de Lausanne, 1015 Lausanne, Switzerland

Charge transfer complexes and charged radical $\pi$-dimers span the field of organic electronics, making their characterization of considerable interest. The electronic structure of charge transfer complexes is highly challenging. ${ }^{1}$ We demonstrate that standard density functionals fail to accurately describe interaction energies of charge-transfer complexes not only because of the missing long-range exchange, as generally assumed, but predominantly because of the ubiquitously present, yet neglected dispersion interactions. ${ }^{2}$ These assertions are based on the evaluation of the interaction energies using DFT coupled with our recent density-dependent dispersion correction (dDsC) $)^{3}$ and high-level $a b$ initio computations. While global hybrid functionals describe well the electronic structure of neutral chargetransfer complexes, long-range corrected exchange is required for the correct qualitative potential energy curve of cationic $\pi$-dimers. Accounting for dispersion via $\mathrm{dDsC}$ is strongly recommended in all cases. Our analysis reflects the delicate error cancellation between the delocalization error (overestimation of charge-transfer) and the missing long-range interactions.
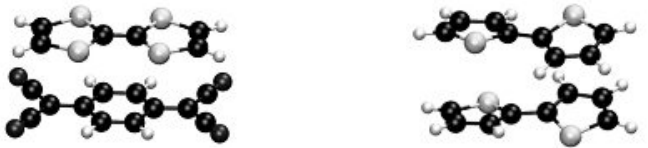

[1] E. Ruiz, D.R. Salahub, A. Vela, J. Am. Chem. Soc. 1995, 117, 1141.

[2] S.N. Steinmann, C. Piemontesi, A. Delachat, C. Corminboeuf, J. Chem. Theory Comput., doi 10.1021/ct200930x.

[3] S.N. Steinmann, C. Corminboeuf, J. Chem. Theory Comput. 2011, 7, 3567 .

Computational Chemistry, Poster Talk

162

DFT studies of HNCO adsorption at the stabilized iron complexes in the ZSM-5 pore

I. Czekaj, S. Brandenberger, O. Kröcher

Paul Scherrer Institute, Villigen, Switzerland izabela.czekaj@psi.ch

The stabilization of monomeric and dimeric iron complexes [1], such as Fe$\mathrm{OH}$ and $\mathrm{HO}-\mathrm{Fe}-\mathrm{O}-\mathrm{Fe}-\mathrm{OH}$ and $\mathrm{Fe}-\mathrm{O}-\mathrm{Fe}$, in the $\mathrm{ZSM}-5$ pore has been investigated using a DFT approach with a cluster model. A $\mathrm{Si}_{20} \mathrm{O}_{53} \mathrm{H}_{28}$ cluster was chosen to represent part of a single channel in the ZSM-5. In this basic cluster, two Si atoms were replaced by two aluminum atoms to simulate the exchange sites in the ZSM-5 and to determine the energetically favorable locations for Al-Al couples. Inside the clusters of the ZSM-5 structure, iron oxohydroxide and oxide species formed stable monomer and dimmer configurations. In both cases, the iron sites bound to the oxygen atoms of $\mathrm{AlO}_{4}^{-}$; this occurred because $\mathrm{AlO}_{4}^{-}$tetrahedra carry greater negative charges and attract iron cations stronger than $\mathrm{SiO}_{4}$ tetrahedra. The stabilization energy per iron site was about $3.5 \mathrm{eV}$ for the monomeric iron complex and $1.0 \mathrm{eV}$ to $2.5 \mathrm{eV}$ for the different dimeric iron sites. These similar energy levels, in combination with a detailed study of the geometric structure of the iron and oxygen centers in the zeolite, support our hypotheses that the distribution of iron sites follows the distribution of the $\mathrm{Al}$ atoms and that no preferred configurations exist for these interactions. Additionally molecular and dissociative adsorption of isocyanic acid at iron particles has been investigated. The results of theoretical vibration frequencies have been compared with experimental DRIFT data [2].

[1] S. Brandenberger, O. Kröcher, A. Tissler, R. Althoff, Appl. Catal. A 2010, 373, 168 .

[2] G. Piazzesi, D.Nicosia, M. Devadas, O. Kröcher, M. Elsener, A. Wokaun, Catal. Lett. 2007, 115, 33. 
Computational Chemistry, Poster Talk

Atomic Multipole Moments for organic molecules: Reference axis systems and benefits over point charges

\section{Christian Kramer, ${ }^{1,2}$ Peter Gedeck, ${ }^{1}$ Markus Meuwly ${ }^{2}$}

\author{
${ }^{1}$ Novartis Institutes for BioMedical Research, Campus St. Johann, \\ 4056 Basel, Switzerland \\ ${ }^{2}$ Department of Chemistry, University of Basel, Klingelbergstrasse80,
} 4056 Basel, Switzerland

Atomic multipole moments represent a viable option for improving the standard terms used for intermolecular interactions in current biomolecular force fields. Since atomic multipoles are anisotropic by nature, local reference axis systems have to be introduced to align and rotate multipole moments in molecular dynamics. We show how atomic multipoles (MTPs) can be rigorously implemented into common biomolecular force fields. For this, a comprehensive set of local reference axis systems is introduced which represents a universal solution for treating atom-centered multipoles for all small organic molecules and proteins.

We further show that atomic multipoles can reduce the error in reproducing the electrostatic potential (ESP) by $50-90 \%$ compared to point charges Also, the reproduction of the ESP can be dramatically improved by directly fitting the multipoles to the ESP compared to the standard methods of partitioning the electron density and calculating multipole moments for atom-assigned electron densities. Additionally, for flexible molecules it is shown, that conformationally independent multipole moments can be found that acceptably reproduce the ESP. However, these multipole moments can neither be obtained from single conformations or as average of parameters fitted to individual molecules - they have to be fitted to the conformational ensemble.

[1] Kramer, C.; Gedeck, P.; Meuwly, M. Atomic Multipoles: Electrostatic Potential Fit, Local Reference Axis Systems and Conformational Dependence. J. Comp. Chem. accepted.

Computational Chemistry, Talk

CO transfer in the binuclear $a_{3}$ site of cytochrome c oxidase

\section{Maksym Soloviov, Markus Meuwly^}

Department of Chemistry, University of Basel, Klingelbergstrasse 80, $\mathrm{CH}-4056$ Base

Cytochrome c oxidase $(\mathrm{CcO})$ is a protein involved in catalysis of the terminal step in cellular respiration in mitochondria or bacteria [1]. In fact, the cytochrome heme $\mathrm{a}_{3}$ and $\mathrm{Cu}_{B}$ form a binuclear center that is the site of oxygen reduction. In contrast to $\mathrm{O}_{2}, \mathrm{CO}$ forms a stable complex with the enzyme. Due to a greater affinity of $\mathrm{CO}$ for $\mathrm{Cu}_{B}$ than for $\mathrm{Fe}$ (II) of heme $\mathrm{a}_{3}, \mathrm{CO}$ binds to $\mathrm{Cu}_{B}$ when $\mathrm{Fe}-\mathrm{CO}$ bond is broken. As the $\mathrm{Fe}-\mathrm{C}$ and $\mathrm{CO}$ vibrations are only weakly coupled, the $\mathrm{Fe}-\mathrm{C}$ bond can only be broken by electronic excitation $[2,3]$. Experimentally, arrival of $\mathrm{CO}$ to $\mathrm{Cu}_{B}$ site is observed within 2 ps after UV excitation [2].

Such time scales are ideal for atomistic simulations which are capable of following in detail the trajectories of all the atoms involved. There are several possible mechanisms for the transfer of $\mathrm{CO}$ from $\mathrm{Fe}$ (II) to $\mathrm{Cu}_{B}$ : elongation of $\mathrm{Fe}-\mathrm{CO}$ followed by rotation, by forming an intermediate $\mathrm{Cu}_{B}$-OC state or by forming free $\mathrm{CO}$. To obtain an atomistically detailed mechanism and the dynamics of the process, potential energy surfaces (PES) have been build based on DFT calculations, the B3LYP functional and LANL2DZ (for $\mathrm{Cu}$ and $\mathrm{Fe}$ ) and $6-31 \mathrm{G}++$ basis sets have been used. The effective potential has been implemented in CHARMM and MD simulations of CO were carried out to study possible scenarios. The dynamics of $\mathrm{CO}$ bound and unbound to $\mathrm{Fe}$ of heme is analyzed in detail.

[1] J. Koepke, E. Olkhova, Biochimica et Bioph. Acta 2009, 1787, 635-645.

[2] J. Treuffet, K.J. Kubarych et al Proc. Natl. Acad. Sci. 2007, 104, 1570515710

[3] P.A.Anfinrud, C.Han, R.M.Hochstrasser, Proc. Natl. Acad. Sci. 1989, $86,8387-8391$
Computational Chemistry, Poster Talk

\section{The influence of heme doming on the rebinding of NO to Myoglobin}

\author{
Jaroslaw J. Szymczak and Markus Meuwly*
}

Chemistry Department, University of Basel, Klingelbergstrasse 80, 4056 Basel

Recent time-resolved resonance Raman experiments suggested that the photodissociated NO can rebind to the domed, Fe-out-of-plane conformation of the heme unit[1]. This experiment found that the heme-iron motion and NO rebinding kinetics are not synchronized. Upon NO-photolysis, the transition from an in-plane to a domed-Fe conformation occurs on the subpicosecond time scale whereas the opposite process (domed to in-plane) takes $\approx 30$ ps. On the other hand, in the infrared the rapid rebinding component occurs within 5 ps which suggests that the returning ligand can rebind to a domediron conformation, which has already been suggested by Ionascu et al.[2]. To shed additional light on the structural dynamics underlying this process, an extended model for the heme-NO interaction is currently being developed. Apart from the previously considered[3] relative orientation of the NO ligand to the heme-iron, the iron-out-of-plane (doming) coordinate will be explicitly considered in the newly-designed molecular dynamics simulations. The result of performed simulations will help to understand how heme doming affects rebinding of NO to Myoglobin.

[1] S. G. Kruglik and B.-K. Yoo and S. Franzen and M. H. Vos and J.-L. Martin and M. Negrerie PNAS 2010, 107, 1367-136838.

[2] D. Ionascu and F. Gruia and X. Ye and A. Yu and F. Rosca and C. Beck A Demidov and J. S. Olson and P. M. Champion, JACS 2005, 127, 1692116934.

[3] D. R. Nutt, D. R. and M. Karplus, and M. Meuwly, J. Phys. Chem. B 2005, 109, 21118

Endocyclic Cleavage-Induced Anomerization Reactions of Glycosides: QM/MM Molecular Dynamics Simulation Study

\section{Hiroko Satoh $^{1}$, Teodoro Laino ${ }^{2}$, Jürg Hutter ${ }^{3}$}

${ }^{1}$ National Institute of Informatics (NII), Tokyo, 101-8430, Japan, ${ }^{2}$ IBM Research Zürich, 8803 Rüschlikon, ${ }^{3}$ University of Zürich, 8057 Zürich

Pyranosides carrying 2,3-trans-carbamate easily undergo endocyclic cleavage-induced anomerization from the $\beta$-form to the $\alpha$-form in the presence of a weak Lewis acid, despite of the fact that the endocyclic cleavage is assumed to be a minor cleavage mode of glycosides. Manabe reported experimental evidence that confirms the existance of endocleavage in the class of compounds [1]. With DFT calculations as well as further experiments, we and coworkers concluded that, for glycosides with 2,3-trans cyclic protecting group, inner strain caused by the fused rings is the primary factor enhancing the endocleavage reaction [2]. We performed ab initio QM/MM molecular dynamics simulations of the anomerization reaction to gain a better understanding of the reaction mechanism in solution as well as conformational details along the reaction pathway. The simulations demonstrate unequivocally that the reaction path is characterized by peculiar conformational changes promoting the endocyclic way. We will report here the first results of the simulation study.

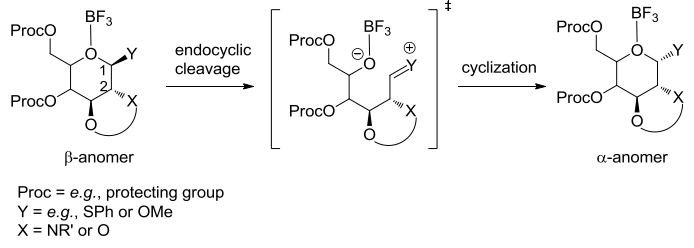

[1] Manabe, S.; Ishii, K.; Hashizume, D.; Koshino, H.; Ito, Y. Chem Eur. J. 2009, 15, 6894.

[2] Satoh, H.; Manabe, S.; Ito, Y.; Lüthi, H. P.; Laino, T.; Hutter, J. J. Am. Chem. Soc. 2011, 133, 5610. 
Computational Chemistry, Talk

Intramolecular Symmetry-Adapted Perturbation Theory

Jérôme F. Gonthier, Clémence Corminbœuf

Laboratory of Computational Molecular Design, Institut des Sciences et Ingénierie Chimiques, Ecole Polytechnique Fédérale de Lausanne Lausanne, CH-1015, Switzerland

Intermolecular interactions play an ubiquitous role in chemistry and biology, with representative examples including protein-ligand interactions, supramolecular assembly, pre-reactive complexes, etc. The theoretical decomposition of intermolecular interactions into physically meaningful terms enhances our understanding of such processes. Among the various existing decomposition schemes, Symmetry-Adapted Perturbation Theory (SAPT) [1] may be considered as the most successful, as it naturally decomposes the interaction energy into physical and intuitive terms such as electrostatics, exchange, dispersion and induction.

On the other hand, the analyses of intramolecular noncovalent interactions, which are responsible for phenomenon such as intramolecular charge transfer (e.g. transannular interactions), conformational and isomer energy differences as well as enzymatic activity are theoretically more challenging to assess, as no energy decomposition scheme is available to date for such interactions.

The presented theoretical scheme fills the gap and proposes an intramolecular variant of the SAPT formalism, which enables the analysis of intramolecular interactions.

The suggested formalism is based on a zeroth-order reference energy state, corresponding to an artificial molecular state in which no interaction is presen between the two fragments of interest. The orbitals are absolutely localized on the fragments and optimized for this state prior to the construction of a suitable zeroth-order wavefunction, from which the different energetic terms of SAPT can be derived.

[1] Jeziorski, B.; Moszynski, R.; Szalewicz, K., Chem. Rev. 1994, 94, 1887.

Computational Chemistry, Talk

Understanding the impact of entropy on the structure and properties of $\mathrm{ScFeO}_{3}$ from first principle simulations

$\underline{\text { Florian Schiffmann }}^{1,2}$, Furio Cora ${ }^{2}$, Ben Slater ${ }^{2}$

${ }^{1}$ ETH Zürich/Department of Materials, ETH Zürich, HIT G 33.2, Wolfgang-Pauli-Strasse 27, 8093 Zürich, Switzerland ${ }^{2}$ UCL London/ Department of Chemistry, University College London, 20 Gordon Street,London WC1H 0AJ

Combining long-range magnetic order with polarity in the same structure is a prerequisite for the design of (magnetoelectric) multiferroic materials. Retaining magnetic order above room temperature still remains a difficult target Recently, $\mathrm{Li}$ et al. presented a polar corundum phase of $\mathrm{FeScO}_{3}$ exposing a weak ferromagnetic ground state up to $356 \mathrm{~K}$. The obtained material shows extended disorder, which prevents a detailed insight in the microscopic structure and properties by experimental methods.

In this study we will present insight from computational chemistry which gives a more detailed understanding of the material. In the first step, we will use results from hybrid to show how the stability of the material is effected by entropy. Afterwards, these results will be used to derive an extended Ising model which is used in Monte-Carlo simulations. The results of these simulations reveal a strong interconnection between the magnetic structure, entropy and polarization in the material.

[1] MR Li, U Adem, SRC. McMitchell, Z Xu, CI Thomas, JE Warren, DV Giap, H Niu, X Wan, RG Palgrave, F Schiffmann, F Cora, B Slater, TL Burnett, MG Cain, AM Abakumov, G van Tendeloo, MF Thomas, MJ Rosseinsky, and JB Claridge, JACS 2012, 134(8), 3737
Computational Chemistry, Talk

Force Matching and Iterative Boltzmann Inversion to Improve DFTB Parameters for Liquid Water

\section{$\underline{\text { M. Doemer }}^{1}$, J. Knaup ${ }^{2}$, I. Tavernelli ${ }^{1}$, U. Rothlisberger ${ }^{1}$}

\author{
${ }^{1}$ Ecole Polytechnique Fédérale, Lausanne, Switzerland
} ${ }^{2}$ Universität Bremen - BCCMS, Bremen, Germany

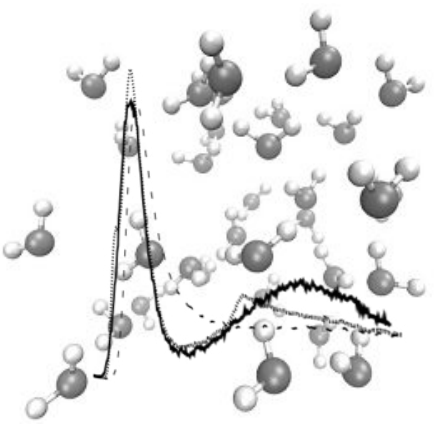

The parameterization of accurate and transferable repulsive potentials is a key ingredient for the density functional tight-binding method (DFTB). In the conventional parameterization scheme the balanced description of different chemical environments involves significant human effort and chemical intuition. The present work aims at an in situ parameterization scheme with limited transferability but maximal accuracy for the chemical and physical environment under investigation. In a comparative study we tested the performance and practical features of two alternative schemes: Force matching and the iterative Boltzmann inversion method. We applied the two schemes to liquid water at ambient conditions, a pathological case for conventional DFTB. Our newly determined parameters significantly improved the structural properties of liquid water.

Computational Chemistry, Talk

A Morphological Study of Magnetite Nanoparticles: influence of the structure on the magnetic dead layer

$\underline{\text { Andrea Barbiero }}^{1}$, Alke Fink $^{1,2}$, Claude Daul ${ }^{1}$

${ }^{1}$ University of Fribourg, Chemin du Musée 9, 1700 Fribourg

${ }^{2}$ Adolph Merkle Institute, Route de l'ancienne Papeterie CP 209, CH-1723 Marly 1, Fribourg

Nanomaterials composed by oxides of transition elements possess interesting and tunable properties that justify the synthetic, analytical and theoretical efforts of the last years, towards the comprehension of their nature. Objects between molecular and bulk state, they require great open-mindedness to treat both from the experimental and the computational [1] point of view. Our research is focused on modeling $\mathrm{Fe}_{3} \mathrm{O}_{4}$ magnetite nanoparticles, which, as Superparamagnetic Iron Oxide Nanoparticles, are interesting for several applications, notably in the bio-medical field [2]. The empirical interatomic potentials used in this study [3], give the possibility to model particles of thousands of atoms and to do Molecular Dynamics simulations of the effect of the solvent on the structure. Our scope is to study, through structural and magnetic calculations, the relationship between the morphology of particles (diameter, surface ligands) and the so called 'magnetic dead layer', also present on other nanoparticles[4]: a surface region of not defined depth, which gives null or very small contribution to the macroscopic magnetization.

[1] Bromley S. T., Moreira I. de P. R.,Neymanab K. M., Illas F., Chem. Soc. Rev., 2009, 38, 2657-2670.

[2] Laurent S., Forge D., Port M., Roch A., Robic C., Vander Elst L., Muller R. N., Chemical Reviews, 2008, 108(6), 2064-2110; Petri-Fink, A., Hofmann, H., IEEE Transactions on NanoBioscience, 2007, 6(4), 289-297. [3] ReaxFF from ADF program; Aryanpour M., van Duin A.C.T., Kubicki J.D., Journal of Physical Chemistry A, 2010, 114, 6298-6307

[4] Curiale J., Granada M., Troiani H. E., Sánchez R. D., Leyva A. G., Levy P., Samwer K., Appl. Phys. Lett., 2009, 95, 04310 
Computational Chemistry, Talk

First-principles structure prediction of $\mathrm{LiBH}_{\mathbf{x}}, x=2$

$$
\underline{\text { Riccarda Caputo }}^{1}
$$

${ }^{1}$ ETH Zürich, Wolfgang-Pauli Strasse 10, CH-8093 Zürich

The partial thermal decomposition of $\mathrm{LiBH}_{4}(\mathrm{~s})$, with evolution of $\mathrm{H}_{2}(\mathrm{~g})$, might lead to the formation of a ternary phase formed by lithium-boronhydrogen, with a stoichiometry $\mathrm{LiBH}_{\mathrm{x}}, x<4$, as computationally suggested [1]. Therein the proposed crystal structures for $x=2.5$ and $x=2.0$ were characterized by the formation of boron-hydrogen anionic chains, ${ }_{\infty}^{1}\left[\mathrm{BH}_{2}\right]$. We extended our investigation of the phase space of the ternary system Li-B$\mathrm{H}$, searching for other possible stable configurations, in particular for $x=2.0$. The general methodological approach combines cluster modelling, simulated annealing (contributed by Prof Adem Tekin, Istanbul Technical University, Informatics Institute, Istanbul, Turkey) and density functional total energy calculations, as successfully applied in structure prediction of complex borohydrides $[2,3,4]$. In addition to chain-structures, ring-structures, in particular with butterfly and square topologies, are found to be local minima with lattice stability ascertained by phonon calculations. The enthalpies of formation, the calculated XRD patterns and the IR spectra are reported for stable structures representative of found family groups: monoclinic, orthorhombic and tetragonal.

[1] R. Caputo, et al., Mol. Phys. 2010, 108, 1236.

[2] R. Caputo, A. Tekin, et al., Chem. Phys. Lett. 2009, 480, 203.

[3] A. Tekin, R. Caputo, Phys. Rev. Lett. 2010, 104, 215501.

[4] R. Caputo, A. Tekin, J. Solid State Chem. 2011, 184, 1622.
Computational Chemistry

DFT Modelling of Ru/Active Carbon Catalyst

I. Czekaj, J. Wambach, F. Vogel

Paul Scherrer Institute, Villigen, Switzerland izabela.czekaj@psi.ch

Ruthenium/active carbon catalyst is used for Haber-Bosch ammonia synthesis as well as supercritical-water gasification of wet biomass for methanation processes. Both reactions are structure sensitive and requires specific active sites at the catalyst. It has been found experimentally that mainly B5 sites are present and actively taking part in catalysis [1]

The main task of our DFT studies is finding stable Ru nanoparticle at graphite using cluster model. Different Ru nanoparticles have been tested starting from 3 up to 17 atoms. The graphite has been modeled by single and double layer. The epitaxial growth of $\mathrm{Ru}$ at graphite follows two locations [2]. However our DFT results suggest that only one of them is stable. Basing on such preliminary results we were able to build and stabilize at carbon support $\mathrm{Ru}$ nanoparticle with three B5 active sites. Such a C-supported Runanoparticle with B5-sites gives potential for further molecule adsorption and reaction mechanism studies. The all investigated structures and configurations for different Ru-nanoparticles will be presented.

[1] S.B. Vendelbo, M. Johansson, J.H. Nielsen, Ib Chorkendorff Phys. Chem. Chem. Phys. 2011, 13, 10333.

[2] Z. Song et al., J. Am. Chem. Soc. 2004, 126, 8576.
Computational Chemistry

How many Chiral Centers can Raman Optical Activity Spectroscopy Distinguish in a Molecule?

Benjamin Simmen, Thomas Weymuth, Markus Reiher ${ }^{1}$

${ }^{1}$ ETH Zürich, Laboratorium für Physikalische Chemie, Wolfgang-Pauli-Str. 10, 8093 Zürich, Switzerland

To study the capabilities and limitations of Raman Optical Activity, (-)$(M) \sigma-[10]$ Helicene and $(-)-(M) \sigma-[4]$ Helicene serve as scaffold molecules on which new chiral centers are introduced by substitution of hydrogen atoms with other functional groups. These functional groups are deuterium atoms, fluorine atoms, and methyl groups. Multiply deuterated species are compared. Then, results of singly deuterated derivatives are compared against results obtained from singly fluorinated and methylated derivatives. The analysis required the calculation of a total of 2433 Raman Optical Activity spectra. The method we propose for the comparison of the various Raman Optical Activity spectra is based on the total intensity of squared difference spectra. This allows a qualitative comparison of pairs of Raman Optical Activity spectra and the extraction of the pair of most similar Raman Optical Activity spectra for each group of stereoisomers. Different factors were accounted for, such as the spectral resolution (modeled by line broadening) and the range of vibrational frequencies considered. In the case of $\sigma$-[4]Helicene all generated stereoisomers in each group can be distinguished from one another by Raman Optical Activity spectroscopy. For $\sigma$-[10]Helicene this holds except for the lower one of the two resolutions considered. Here, the group consisting of stereoisomers with five chiral centers contains at least one pair of derivatives whose Raman Optical Activity spectra cannot be distinguished from one another. This indicates that an increased molecular size has a negative effect on the number of chiral centers which can be distinguished by Raman Optical Activity spectroscopy. Regarding the different substituents, stereoisomers are distinguishable in Raman Optical Activity spectroscopy the better, the more distinct the signals of the substituent are from the rest of the spectrum.
Computational Chemistry

MoVIPAC:Vibrational Spectroscopy with a Robust Meta-Program for Massively Parallel Standard and Inverse Calculations

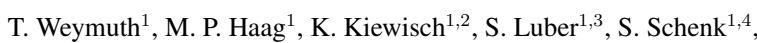
Ch. R. Jacob ${ }^{5}$, C. Herrmann ${ }^{6}$, J. Neugebauer ${ }^{7}$, M. Reiher ${ }^{1}$

${ }^{1}$ ETH Zurich, Wolfgang-Pauli-Str. 10, 8093 Zürich, CH

${ }^{2}$ VU University Amsterdam, De Boelelaan 1083, 1081 HV Amsterdam, NL

${ }^{3}$ Yale University, P.O. Box 208107, New Haven, CT 06520-8107, USA

${ }^{4}$ BASF SE, B001, 67056 Ludwigshafen, DE

${ }^{5}$ KIT, Wolfgang-Gaede-Str. 1a, 76131 Karlsruhe, DE

${ }^{6}$ University of Hamburg, Martin-Luther-King-Platz 6, 20146 Hamburg, DE

${ }^{7}$ Technical University Braunschweig, Hans-Sommer-Str. 10, 38106 Braunschweig, DE

The calculation of vibrational spectra for large molecules is a challenge, as the computational cost increases sharply with system size. The program package MOVIPAC [1] is designed for such massively parallelized calculations implementing efficient subsystem approaches such as Mode-Tracking [2], IntensityTracking [3], and the Cartesian Tensor Transfer Method [4,5] which allow for a considerable decrease in computational cost while offering the desired properties at high accuracy. MOVIPAC unites improved and extended versions of the programs SNF [6] and AKIRA [2] yielding a comprehensive tool for the calculation of vibrational spectra. As the analysis of such spectra is challenging due to the huge amount of data, MoVIPAC includes the LoCVIB package for interpreting the data in an intuitive way employing localized modes [7].

[1] T. Weymuth, et al., J. Comput. Chem. 2012, submitted.

[2] M. Reiher, J. Neugebauer, J. Chem. Phys. 2003, 118, 1634.

[3] K. Kiewisch, et al., Chimia 2009, 63, 270.

[4] P. Bouř, et al., J. Comput. Chem. 1997, 18, 646.

[5] N. S. Bieler, et al., J. Chem. Theory Comput. 2011, 7, 1867.

[6] J. Neugebauer, et al., J. Comput. Chem. 2002, 23, 895.

[7] Ch. R. Jacob, M. Reiher, J. Chem. Phys. 2009, 130, 084106. 
Computational Chemistry

\section{Analysis of the Cartesian Tensor Transfer Method for Calculating Vibrational Spectra of Polypeptides}

\section{Moritz P. Haag ${ }^{1}$, Noah S. Bieler ${ }^{1}$, Christoph R. Jacob ${ }^{2}$, Markus Reiher ${ }^{1}$}

${ }^{1}$ ETH Zurich, Laboratorium für Physikalische Chemie,

Wolfgang-Pauli-Strasse 10, 8093 Zurich, Switzerland

${ }^{2}$ Karlsruhe Institute of Technology (KIT), Center for Functional

Nanaostructures, Wolfgang-Gaede-Str. 1a, 76131 Karlsruhe, Germany

The Cartesian Tensor Transfer Method (CTTM) [1] was proposed as an efficient way to calculate infrared, Raman, and Raman Optical Activity (ROA) spectra for large molecules from the Hessian matrix and property tensor derivatives calculated for smaller molecular fragments. Although this approach has been widely used, its reliability has not been analyzed in depth yet. Especially for ROA spectra, such an analysis became only recently possible because of methodological advances that allow for the calculation of full ROA spectra of fairly large molecules with large basis sets. In this work[2], we investigate an $\alpha$-helical polypeptide of 20 alanine amino acids, for which we reported the full ROA spectra earlier, in order to study the CTTM for protein subunits. By comparing the full first-principles calculation of the vibrational spectra with spectra reconstructed with the CTTM from different fragment sizes, we find that infrared and Raman spectra are mostly well reproduced. However, this is not the case for the ROA spectrum. The results of this work might have implications for peptide and protein CTTM ROA spectra that have already been published in the literature.

[1] P. Bouř, J. Sopková, L. Bednárová, P. Maloň, T. A. Keiderling, J. Comput Chem. 1997, 18, 646.

[2] N. S. Bieler, M. P. Haag, C. R. Jacob, M. Reiher, J. Chem. Theory Comput. 2011, 7, 1867 .

Computational Chemistry

Accurate $a b$ initio spin densities

$\underline{\text { Katharina Boguslawski }^{1}}$, Konrad H. Marti ${ }^{1}$, Örs Legeza ${ }^{2}$, Markus Reiher ${ }^{1}$

${ }^{1}$ ETH Zurich, Laboratorium für Physikalische Chemie,

Wolfgang-Pauli-Strasse 10, 8093 Zurich, Switzerland

${ }^{2}$ Wigner Research Centre, P.O Box 49, H-1525 Budapest, Hungary

For large molecular systems such as transition metal complexes, densityfunctional theory (DFT) became instrumental in theoretical studies of mechanisms in metal-mediated catalysis. Yet, the treatment of open-shell systems remains a challenge for DFT [1]. In addition to the difficult prediction of ground states from states of different spin, spin density distributions considerably depend on the approximate exchange-correlation density functional if transition metal complexes containing noninnocent ligands are considered Especially, nitric oxide as an open-shell molecule endows a corresponding transition metal nitrosyl complex with a complicated electronic structure for which qualitatively correct spin densities are difficult to obtain within the standard Kohn-Sham formalism [2].

We present highly accurate spin densities for an iron nitrosyl complex which we have identified as a challenging system for DFT and standard electron correlation methods [3]. Our approach is based on the density-matrix renormalization group (DMRG) algorithm [4] and allows the treatment of active orbital spaces beyond the current limit of 18 electrons correlated in 18 molecular orbitals. Furthermore, the explicit reconstruction of a complete-activespace configuration-interaction-type wave function [5] provides insights into chemically interesting features of the studied molecules such as the distribution of $\alpha$ - and $\beta$-electrons in terms of Slater determinants, CI coefficients, and natural orbitals. The DMRG reference spin densities can be used to validate the accuracy of DFT spin densities and assess the quality of the restricted active orbital spaces.

M. Reiher, Chimia 2009 63, 140.
K. Boguslawski, C. R. Jacob and M. Reiher, J. Chem. Theory Comput. 2011, 7, 2740.

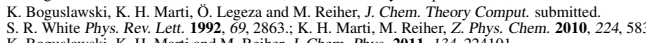

Computational Chemistry

Exact decoupling of the relativistic Fock operator

$$
\underline{\text { Daoling Peng }^{1}, \text { Markus Reiher }^{1}}
$$

${ }^{1}$ Laboratorium für Physikalische Chemie, ETH Zürich, Wolfgang-Pauli-Strasse 10, CH-8093 Zurich, Switzerland

It is generally acknowledged that the inclusion of relativistic effects is crucial for the theoretical description of heavy-element-containing molecules. Four-component Dirac-operator-based methods serve as the relativistic reference for molecules and highly accurate results can be obtained - provided that a suitable approximation for the electronic wave function is employed. However, four-component methods applied in a straightforward manner suffer from high computational cost and the presence of pathologic negative-energy solutions. To remove these drawbacks, a relativistic electron-only theory is desirable for which the relativistic Fock operator needs to be exactly decoupled. Recent developments in the field of relativistic two-component methods demonstrated that exact decoupling can be achieved following different strategies. Three different approaches - DKH [1], X2C [2], and BSS [3] exist for this purpose and they are all intimately related. The theoretical formalism of these exact-decoupling approaches is reviewed in this presentation followed by a comparison of efficiency and results [4]. A local approach for these exact-decoupling methods is proposed to reduce the computational cost of large molecules [5].

[1] B. A. Hess, Phys. Rev. A 1986, 33, 3742; M. Reiher and A. Wolf, J. Chem. Phys. 2004, 121, 2037.

[2] W. Kutzelnigg and W. Liu J. Chem. Phys. 2005, 123, 244102; W. Liu and D. Peng J. Chem. Phys. 2006, 125, 044102.

[3] M. Barysz, A. Sadlej and J. G. Snijders Int. J. Quantum Chem. 1997, 65, 225; D. Kȩdziera and M. Barysz Chem. Phys. Lett. 2007, 446, 176.

[4] D. Peng and M. Reiher, Theor. Chem. Acc. 2012, 131, 1081.

[5] D. Peng and M. Reiher, In preparation.

Computational Chemistry

Theoretical Studies into the Mechanism of $[\mathrm{Fe}]$ and $[\mathrm{FeFe}]$ Hydrogenases

\section{Arndt R. Finkelmann ${ }^{1}$, Martin T. Stiebritz ${ }^{1}$, Markus Reiher ${ }^{1}$}

${ }^{1}$ Laboratorium für Physikalische Chemie, ETH Zürich,

Wolfgang-Pauli-Strasse 10, CH-8093 Zürich, Switzerland

Enzymes which catalyze the reversible oxidation or formation of molecular hydrogen are called hydrogenases and grouped into three classes, namely $[\mathrm{FeFe}],[\mathrm{NiFe}]$ and $[\mathrm{Fe}]$ hydrogenases. They are of interest as potential catalysts in renewable energy technologies. $[\mathrm{FeFe}]$ hydrogenases, which have the highest hydrogen formation turnover rates, are irreversibly inhibited by $\mathrm{O}_{2}$ which is a major drawback for their technical applicability [1]. [Fe] hydrogenase is, however, not inhibited by $\mathrm{O}_{2}$.

To relate both active sites, we construct cluster models of $[\mathrm{Fe}]$ and $[\mathrm{FeFe}]$ hydrogenases active sites by mutual ligand exchange [2]. The influence of the first-shell ligand sphere on the catalytic cycle of these models is investigated with density functional theory calculations. Crucial reaction steps of $[\mathrm{Fe}]$ hydrogenase's catalytic mechanism are strongly affected by the change to $[\mathrm{FeFe}]$ hydrogenase-like first-shell ligands [3] and the reactivity is biased towards $[\mathrm{FeFe}]$ hydrogenase-like reactivity. For [FeFe] hydrogenase we present a full reaction mechanism based on a large model of the active site which includes interactions of important residues with the $\mathrm{H}$ cluster. A detailed picture of a possible catalytic cycle provides answers to the question if a bridging or terminal-bound hydride species is the central catalytic intermediate [3].

[1] M.T. Stiebritz, M. Reiher, Chem. Sci. 2012, doi:10.1039/C2SC01112C M.T. Stiebritz, M. Reiher, Inorg. Chem. 2009, 48, 7127; C. Lambertz, N. Leidel, K.G.V. Havelius, J. Noth, P. Chernev, M. Winkler, T. Happe, M. Haumann, J. Biol. Chem. 2011, 286, 40614.

[2] M.T. Stiebritz, M. Reiher, Inorg. Chem. 2010, 49, 5818.

[3] A.R. Finkelmann, M.T. Stiebritz, M. Reiher, in preparation 
Computational Chemistry

\section{Fe-4S Clusters: Reactivity vs. Cluster Geometry}

Maike Bergeler $^{1}$, Martin T. Stiebritz ${ }^{1}$, Markus Reiher ${ }^{1}$

${ }^{1}$ Laboratorium für Physikalische Chemie, ETH Zürich, Wolfgang-Pauli-Str. 10, 8093 Zürich, Switzerland

Iron-sulfur clusters play a key role in electron transfer processes, metabolism, signal transduction and activation of low-reactivity molecules like $\mathrm{N}_{2} / \mathrm{H}_{2}$ [1]. Despite the same type of ligation of the $4 \mathrm{Fe}-4 \mathrm{~S}$ cluster by four cysteines in several proteins, the $4 \mathrm{Fe}-4 \mathrm{~S}$ clusters feature a recognizably different reactivity towards reactive oxygen species (ROS) [2], which can lead to an irreversible damage of the protein $[1,3]$. One reason for the varying reactivities towards ROS could be the effect of the protein environment on the first ligand sphere due to the inflexibility of the protein backbone.

Here, we studied $D_{2 d}$-symmetric cubane type $4 \mathrm{Fe}-4 \mathrm{~S}$ cluster models ligated by four cysteines with constraints on the ligands to mimic the spatial constraints imposed by the protein environment using Density Functional Theory. Spin couplings within the cubane were treated by employing the brokensymmetry approach. We investigated the reactivity towards ROS depending on spin state and symmetry of the cluster by systematic distortions of the geometric constraints. We conclude that both spin coupling and reactivity of $4 \mathrm{Fe}-4 \mathrm{~S}$ clusters are determined by deviations from the ideal cluster geometry as given by the anchoring in the particular protein in which they occur.

[1] J. A. Imlay, Mol. Microbiol. 2006, 59, 1073; I. R. Tsaneva, B. Weiss, J. Bacteriol. 1990, 172, 4197; K. A. Vincent, A. Parkin, O. Lenz, S. P. J. Albracht, J. C. Fontecilla-Camps, R. Cammack, B. Friedrich and F. A. Armstrong, J. Am. Chem. Soc. 2005, 127, 18179.

[2] M. T. Stiebritz and M. Reiher, Chem. Sci. 2012, 10.1039/c2sc01112c.

[3] K. A. Vincent, A. Parkin and F. A. Armstrong, Chem. Rev. 2007, 107, 4366; M. K. Bruska, M. T. Stiebritz and M. Reiher, J. Am. Chem. Soc. 2011, 133, 20588.
Computational Chemistry

Dioxygen induced degradation of $\left[\mathrm{Fe}_{4} \mathrm{~S}_{4}\right]$ cubane clusters: a quantum chemical perspective.

Bruska, M.K. ${ }^{1}$, Stiebritz M.T. ${ }^{1}$, Reiher M. ${ }^{1}$

${ }^{1}$ Laboratory of Physical Chemistry, ETH Zurich, Wolfgang-Pauli-Strasse 10, 8093 Zurich, Switzerland

Lifetimes and decomposition products of the $\left[\mathrm{Fe}_{4} \mathrm{~S}_{4}\right]$ clusters exposed to oxygen range from rapid and complete degradation of the Fe-S cluster for enzymes like [FeFe]-hydrogenases [1] to $\mathrm{O}_{2}$ stable cubanes harbored by the Escherichia coli DinG helicase [2]. A question not fully resolved is how and why some of the solvent accessible Fe-S cubane clusters survive longer in aerobic environment.

Here, we address the problem of oxygen sensitivity of the $\left[\mathrm{Fe}_{4} \mathrm{~S}_{4}\right]$ cubane clusters with density functional theory (DFT) calculations on several $\mathrm{O}_{2}$ addition and decomposition reactions at $\left[\mathrm{Fe}_{4} \mathrm{~S}_{4}\right]$ cubanes. The presented work is a continuation of the research recently published by our group [3] and discusses the general principles of decomposition pathways for the structural model of an isolated $\left[\mathrm{Fe}_{4} \mathrm{~S}_{4}\right]$ cubane and refers them to the study on a 800 atoms model of [FeFe]-hydrogenase active site in order to discuss the influence of a protein environment. In particular, we discuss coordination energetics of dioxygen adducts of the $\left[\mathrm{Fe}_{4} \mathrm{~S}_{4}\right]$ cubane, possibility of ROS formation, transition state structures and activation barriers for the oxygen-induced destruction of the Fe-S cluster, and the role of different spin-coupling schemes at iron centers.

The theoretical investigation of the decomposition pathways of Fe-S cubanes opens a possibility for in silico mutation studies not only for Fe-only hydrogenases but also other enzymes harboring $\left[\mathrm{Fe}_{4} \mathrm{~S}_{4}\right]$ cubanes.

[1] Vincent, K.A. et al., J. Am. Chem. Soc. 2005, 127, 18179.

[2] Ren, B., Duan, X., Ding, H., J. Biol. Chem. 2009, 284, 4829.

[3] Bruska, M.K., Stiebritz, M.T., Reiher, M., J. Am. Chem. Soc. 2011, 133, 20588.
Computational Chemistry

A first-principles study of the redox properties of $\left[\mathrm{Ru}(\mathrm{bpy})_{3}\right]^{2+/ 3+}$

Polydefkis Diamantis, Ivano Tavernelli, Ursula Rothlisberger*

Ecole Polytechnique Fédérale de Lausanne (EPFL), Av. Forel 2, CH-1015 Lausanne, Switzerland

Car-Parrinello molecular dynamics simulations based on DFT/BP86 were performed, in order to study the redox reaction:

$\left[\mathrm{Ru}(\mathrm{bpy})_{3}\right]^{2+} \rightarrow\left[\mathrm{Ru}(\mathrm{bpy})_{3}\right]^{3+}$, for both the singlet and the triplet state.

For the calculation of the redox free energy difference $\left(\Delta A_{R \rightarrow O}\right)$ and the reorganization free energy $(\lambda)$ of both reactions, a well known method was applied [1][2], one that assumes Marcus theory for electron transfer, and in which $\Delta \mathrm{A}_{\mathrm{R} \rightarrow \mathrm{O}}=\langle\Delta \mathrm{E}\rangle_{\mathrm{R}}+\langle\Delta \mathrm{E}\rangle_{\mathrm{O}}$ (the average ionization potential of the reduced state and the average electron affinity of the oxidized state respectively). The method provided reasonable results for both $\Delta \mathrm{A}_{\mathrm{R} \rightarrow \mathrm{O}}(5.37 \mathrm{eV}$ and $3.60 \mathrm{eV}$ for singlet and triplet states respectively) and $\lambda(1.19 \mathrm{eV}$ singlet and $1.25 \mathrm{eV}$ for singlet and triplet states respectively), in good agreement with existing experimental and calculated values (singlet $\Delta \mathrm{A}_{\mathrm{R} \rightarrow \mathrm{O}}=5.60 \mathrm{eV}$, $\lambda=1.21 \mathrm{eV}$ ) [3]. In order to further check the validity of Marcus Theory, the free energy curves of $\left[\mathrm{Ru}(\mathrm{bpy})_{3}\right]^{2+}$ and $\left[\mathrm{Ru}(\mathrm{bpy})_{3}\right]^{3+}$ were also plotted for both reactions, as functions of $\Delta \mathrm{E}-\Delta \mathrm{A}_{\mathrm{R} \rightarrow \mathrm{O}}$. In both cases, the two free energy curves were parabolas to a very good approximation, a proof that Marcus theory can apparently describe quite accurately these two reactions.

[1] M. Cascella, A. Magistrato, I. Tavernelli, P. Carloni, U. Rothlisberger, Proc. Natl. Acad. Sci. USA, 2006, 102, 19641

[2] J. Blumberger, I. Tavernelli, M.L. Klein, M. Sprik, J. Chem. Phys., 2006, 124,64507

[3] R. Seidel, M. Faubel, B. Winter, J. Blumberger, J. Am. Chem. Soc., 2009, 131, 16127
Computational Chemistry

Perfomance of Dispersion-Corrected Atom-Centered Potentials: Non-equilibrium gas phase geometries and condensed matter systems.

\section{A. Laktionov, M. Doemer, I. Tavernelli, and U. Rothlisberger}

Ecole Polytechnique Fédérale de Lausanne, Lausanne, Switzerland

Van-der-Waals interactions play a crucial role in biological and pharmaceutically relevant systems. Unfortunately, standard density functional theory (DFT) calculations within generalized gradient approximations (GGA) fail to account for these weak interactions. In recent works, dispersion-corrected atom-centered potentials (DCACPs) have been developed to account for longrange dispersion forces between molecules within a DFT/GGA framework. DCACP are based on the idea of representing a non-local dispersion interaction potential as a sum of atom-centered semilocal potentials.

$$
\begin{gathered}
v_{\mathrm{xc}}^{\text {extended }}=v_{\mathrm{xc}}+\sum_{\mathrm{I}} v_{\mathrm{I}}^{\mathrm{DCACP}}\left(\mathbf{r}, \mathbf{r}^{\prime}\right) \\
v_{I}^{\mathrm{DCACP}}\left(\mathbf{r}, \mathbf{r}^{\prime}\right)=\sum_{\ell=0}^{\ell_{\max }} \sum_{m=-\ell}^{+\ell} Y_{\ell m}^{I}(\hat{\mathbf{r}}) p_{\ell}^{I}(r) \sigma_{1} p_{\ell}^{I}\left(r^{\prime}\right) Y_{\ell m}^{I *}\left(\hat{\mathbf{r}}^{\prime}\right)
\end{gathered}
$$

where $r=r-R_{I}$ is the distance from the electron to the position of nucleus $I$, and $p_{\ell}^{I}(r)$ is the normalized projector, embraced by spherical harmonics. Such a choice of sets of functions has several advantages. First, separating variables reduces the computational cost of the non-local dispersion term from $N^{6}$ to $N^{3}$. Second, the exponential decay of projectors allows to introduce a cutoff on the radial grid, making calculations cheaper. Thirdly, as DCACP use only unoccupied channels in the Goedecker-Teter-Hutter (GTH) pseudopotentials, there is no necessity to modify the code, when planewave calculations are performed. Within this approach dispersion interactions can now be accurately described by DFT at the cost of roughly a standard GGA functional.

Here, we demonstrate that by adding more terms to the expansion (2) one can retrieve the correct $R^{-6}$ asymptotics of the tail of the interaction curve. Additionally, we present results on the physisorption of organic molecules on gold and silicon surfaces and on the influence of halogen bonding on the molecular structure in gas-phase clusters up to molecular crystals. 
Computational Chemistry

Ab Initio Based Methodologies for Investigation of Charge Transfer

\section{Through Organic Molecules}

Laura Berstis $^{1}$, Kim Baldridge $^{1}$

${ }^{1}$ University of Zürich, Winterthurerstrasse 190, 8057 Zürich

The study of charge transfer (CT) reactivity through organic molecules presents vast opportunities to understand and design materials with desirable electronic properties. In the current work, a first principles methodology has been developed for the evaluation of CT properties in nonadiabatic systems, and implemented into the widely used $a b$ initio computational suite GAMESS. In this implementation a broad range of levels of theory, including semiempirical, Hartree-Fock, DFT, or MP2, may be applied to the system studied, to achieve the highest possible accuracy for the size and complexity of the CT system at hand. The calculation of the electronic coupling between an electron donor and acceptor utilizes a localized molecular orbital effective Hamiltonian ${ }^{1}$, relating the coupling between the donor and acceptor through the remaining bridge localized orbitals. Furthermore the possible tunneling pathways available are calculated by means of a Greens function matrix of the bridge localized atomic or molecular orbitals. ${ }^{2}$ These evaluations shed new light on discussions of through-bond and through-space coupling effects. The electronic coupling and pathway analyses enable a quantification of the magnitude of through-space or through-bond coupling, and clarify through which molecular orbitals these interactions are made possible. Additionally the impact of solvent to these CT characteristics is considered in the present work. Through these investigations, an understanding is gained of the impacts of structure, and presence of functional groups on the donor, acceptor or bridge, on the probability and mechanism of the CT reactions, contributing greatly towards the design of materials, which will offer desirable properties.

[1] Teklos A, Skourtis S.S. 2006, J. Chem. Phys. 125, 244103.

[2] C. Kobayashi, K. Baldridge, J.N. Onuchic. 2003, J. Chem. Phys. Vol 119, No. 6.

Computational Chemistry

Direct Second-Order Møller-Plesset Perturbation Energy

A New Implementation Based on a Two-Level Parallelization of Integral Computation and Transformation

$\underline{\text { Mauro Del Ben }}^{1}$, Jürg Hutter ${ }^{1}$ and Joost VandeVondele ${ }^{2}$

${ }^{1}$ Institute of Physical Chemistry, University of Zürich

${ }^{2}$ Department of Materials, ETH Zürich

A conventional implementation of the Second-Order Møller-Plesset (MP2) theory consists in three steps: the two-electron integrals calculation in the atomic orbitals (AOs) basis; the integrals transformation into molecular orbitals (MOs) and the integrals contraction into the MP2 energy. The most time demanding step is the integrals transformation, that scales formally $O\left(N^{5}\right)$, while the other two steps scale as $O\left(N^{4}\right)$, where $N$ is the size of the system. Based on this different scaling behavior, a new parallel direct MP2 energy algorithm is presented for which the total number of processes is divided into groups: the occupied orbitals pairs, in the integrals transformation step, are distributed over all processes, while the integrals over AOs are recomputed for each group. In this way, the communication is restricted to the members of the group, avoiding a global message-passing scheme that involves all processes. Test calculations demonstrate scalability up to thousands of processes and ability to handle systems containing thousands of basis functions. The proposed algorithm outperforms other parallel MP2 implementations with computational savig of $50 \%$ achived by tuning the number of processes within a group. The developed algorithm is promising for extending the range of applications of the MP2 method.
Computational Chemistry

Sector model scheme for placement of explicit water molecules for $\mathrm{pKa}$ prediction

Rebecca A Abramson, Kim K Baldridge

Organic Chemistry Institute, University of Zürich, Winterthurerstrasse 190, CH-8057, Zürich Switzerland

Accurate prediction of the properties associated with proton transfer reactions, in particular the acidity dissociation constant $\left(\mathrm{K}_{\mathrm{a}}\right)$, are a benchmark challenge for theory. Considerable efforts have been made in the last decade towards prediction within 0.5 units, ${ }^{1}$ which is the minimum level of accuracy for many problems such as structure based drug design ${ }^{2}$. Hybrid approaches, referred to as continuum cluster (CC), are the most recent and promising development in pKa prediction. ${ }^{1,3,4,5}$ They attempt to capture some of the missing first solvation shell effects by adding a small number $(<4)$ solvent molecules explicitly around the solute embedded in the solvent cavity. However, a number of practical challenges arise in application of this method due to the need to have a consistent framework for determination of optimal number and position of solvent molecules surrounding the solute. We propose a sector model approach to investigate in greater detail the role of explicit first-shell solvation of a set of carboxylic acids and their conjugate bases. This model offers the possibility of systematizing the study of these effects according to the degree of solvation $\left(\mathrm{S}_{D}\right)$ and the configuration of solvation $\left(\mathrm{S}_{C}\right)$, which allows a number of rules for future prediction to be elucidated.

[1] Ho, J., Coote, M.L., 2011, Wires Comput. Mol. Sci., 2011, 1(5), 649660

[2] Klicic, J.J. Friesner, R.A., Liu, S., Guida, W., J. Phys. Chem. A, 2002, 106, 1327-1335

[3] Pliego, J.R., Riveros, J.M., J. Phys. Chem. A, 106, 2002, 7434-7439

[4] Eckert, F., Diedenhofen, M., Klamt, A., Molecular Physics, 2010, 108

(3), 229-241

[5] Kelly, C.P., Cramer, C.J., Truhlar, D.G., J. Phys. Chem. A, 2006, 110 (7), 2493-9

Computational Chemistry

Dielectric constant calculations:

DFT approach using Tree Monte Carlo

$\underline{\text { Mandes Schönherr }}^{1}$, Joost VandeVondele ${ }^{2}$, Jürg Hutter ${ }^{2}$

${ }^{1}$ Institute of Physical Chemistry, University of Zurich

${ }^{2}$ Department of Materials, ETH Zurich

The development of high performance computers significantly increase the computing power available. To use this potential, novel algorithms have to be adopted to the increase of parallelization. In the case of molecular simulations, the scalability of Molecular Dynamics (MD) is ultimately limited, and the step-by-step integration essentially serializes the procedure. We present a Tree Monte Carlo (TMC) algorithm which permits the simultaneous calculation of many molecular configurations. Using a speculative approach, various pathways, accounting for both rejection and acceptance of proposed moves, can be computed in parallel. Key to this approach is the fact that generating configurations for the Monte Carlo algorithm is cheap and can be performed without computing forces. The TMC procedure respects detailed balance, and in fact the sequence of Monte Carlo moves used is independent of the number of simultaneously computed tasks. The parallel efficiency of the TMC algorithm depends on the acceptance probabilities of the moves. Various configurational changes are implemented, varying from standard MC moves, such as atom and molecule translation or molecule rotation, to more intelligent moves such as proton reordering, two potential moves (Nested Monte Carlo), hybrid Monte Carlo, parallel tempering and Nested Monte Carlo. Calculation costs are significantly reduced using approximate potentials. Sub-box methods increase the acceptance rate and hence the efficiency. Further efficiency gains will come through dynamic process termination and acceptance estimation. Using this algorithm, we were able to calculate the dielectric constant, a property with long relaxation time, of ice Ih structures using PBE and PBE0. The PBE model overestimates the dielectric constant compared to experimental values. In contrast to that, the value using PBE0 is in good agreement with the experimental results. The results of both methods were converged. 
Computational Chemistry

\section{Hexagonal Boron Nitride on Nickel and Copper Surfaces}

Ralph Koitz, Marcella Iannuzzi, Ari P. Seitsonen, Jürg Hutter

University of Zurich, Winterthurerstrasse 190, CH-8057 Zürich

Numerous transition metals have been studied as substrates for the adsorption of monolayers of hexagonal boron nitride (h-BN), and promising applications have been suggested for these materials [1]. We studied the structural, electronic, and magnetic properties of h-BN on the (111) surfaces of $\mathrm{Ni}$ and $\mathrm{Cu}$.

While the lattice-matching of h-BN and $\mathrm{Ni}$ is near-perfect, we observe that the adsorption registry plays an important role for the metal-h-BN interaction. Configurations with $\mathrm{N}$ atoms atop $\mathrm{Ni}$ positions are bound with significantly higher energies and smaller interlayer distances. We also find a clear dependence of the surface magnetic moment on the interlayer distance.

$\mathrm{Cu}$ has been studied less extensively as a substrate, but has recently seen increasing interest. Small slabs of $\mathrm{Cu}$ show adsorption characteristics with comparatively little differentiation between surface registries and low adsorption energies. In contrast, larger systems, such as a $24 \times 24$ rotated h-BN layer on a $23 \times 23 \mathrm{Cu}$ cell, form moiré patterns resulting in a structural as well as electronic corrugation of the surface (see figure).

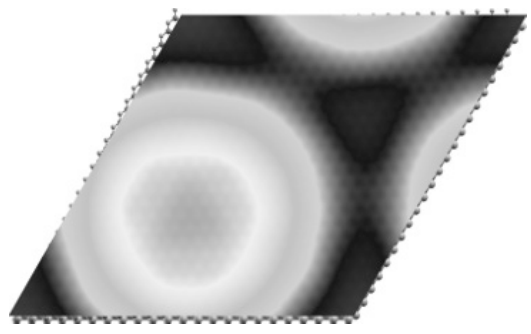

[1] R. Laskowski et al. Phys Rev B 2008, 78, 045409.

Computational Chemistry

Influence of Donor-Acceptor Substitution on the Molecular Properties of Linearly Conjugated Oligomers

Elizabeth Chirackal Varkey ${ }^{1,2}$, Peter Andreas Limacher ${ }^{1}$, Hans Peter Lüthi ${ }^{1}$, Jürg Hutter ${ }^{2}$

${ }^{1}$ Laboratory of Physical Chemistry, ETH Zurich, ${ }^{2}$ Institute of Physical Chemistry, University of Zurich.

We performed a systematic investigation of the impact of different donor-acceptor substitution patterns as a function of backbone type and size. The types of backbones investigated are trans-polyacetylene (PA), polyyne (PY) and polythiophene (PT). All calculations were performed using density functional theory (CAM-B3LYP). To investigate the response of the properties on electron delocalization, we evaluated the BLA, polarizability, hyperpolarizability and rotational barrier. The studies revealed that BLA and rotational barrier follow an exponential behaviour (in terms of chain length and substituents) whereas, polarizabilities and hyperpolarizabilities follow a power law. The Superposition Model [1] was applied to explore the cooperative effect of substituents in both homo and hetero end-capped oligomer chains. We observed that the cooperative effect of BLA, polarizability, hyperpolarizability and rotational barrier decays exponentially as the oligomer chain length, regardless of the donor-acceptor strength.

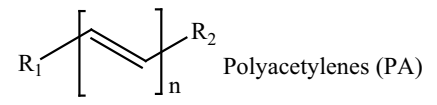

$\mathrm{R}_{1} \rightleftharpoons\left[\overline{\mathrm{n}} \mathrm{R}_{2}\right.$ Polyynes (PY)

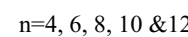

$\mathrm{R}_{1}, \mathrm{R}_{2}=\mathrm{H}, \mathrm{NH}_{2}, \mathrm{NO}_{2}, \mathrm{CN} \& \mathrm{OH}$

Figure 1: Oligomers investigated
[1] S. Borini, P. A. Limacher, H. P. Lüthi, J. Phys. Chem. A, 2010, 114, 2221.
Computational Chemistry

Implementation of the image charge method in QM/MM

Dorothea Golze, Marcella Iannuzzi, Jürg Hutter

University of Zurich, Winterthurerstr. 190, CH-8057 Zurich, Switzerland

A method for including polarization effects within hybrid quantum mechanics/molecular mechanics (QM/MM) simulations of physisorbed adsorbatemetal systems is presented. In a standard QM/MM description, the adsorbed molecules are treated by QM and the metal by MM. The interactions between adsorbate and metal are usually described by a pair potential where electrostatic interactions are neglected. However, polar adsorbates can induce charges in the metal and interact with these charges. This classical phenomenon is referred to as image effect. It was shown that image charges are important for determining the assembling behavior of organic molecules on metal surfaces [1]. Image charges are also needed for a comprehensive description of processes at electrode interfaces [2].

In this work, the image charge method was implemented in the QM/MM module $[3,4]$ of the $\mathrm{CP} 2 \mathrm{~K}$ program package. The implementation is based on the Siepmann-Sprik scheme [5], where the image charge distribution in the metal is modeled by a set of Gaussian charges centered at the metal atoms. The image charges are determined self-consistently by imposing the constantpotential condition within the metal. The image charge method was applied to simple nitro-substituted aromatic molecules at $\mathrm{Au}(111)$ interfaces and the results were compared to full DFT.

[1] G. Tomba et al., ACS Nano 2010, 4, 7545.

[2] S. K. Reed, O. J. Lanning, P. A. Madden, J. Chem. Phys. 2007, 126, 084704.

[3] T. Laino, F. Mohamed, A. Laio, M. Parrinello, J. Chem. Theory Comput. 2005, $1,1176$.

[4] T. Laino, F. Mohamed, A. Laio, M. Parrinello, J. Chem. Theory Comput. 2006, 2, 1370

[5] J. I. Siepmann, M. Sprik, J. Chem. Phys. 1995, 102, 511.

Computational Chemistry

190

Quantum Chemical Investigation of the Solvent Effect on Two Competing Trifluoromethylation Reaction Mechanisms Involving $\lambda^{3}$-Iodane Reagents: At the Limit of the PCM Model?

Oliver Sala, Hans Peter Lüthi, Halua Pinto de Magalhães, Antonio Togni

ETH Zürich, Wolfgang-Pauli-Strasse 10, CH-8093 Zürich, Switzerland

Hypervalent iodines are important reagents for the trifluoromethylation of nucleophiles such as $N$-heterocyclic compounds[1]. Two competing reaction mechanisms for the trifluoromethylation were found based on DFT calculations: a reductive elimination (RE) and an $\mathrm{S}_{N} 2$. The solvent effect on these two competing reaction mechanisms was investigated using a PCM model. Whereas the barrier of the $\mathrm{S}_{N} 2$ reaction pathway is raised by the solvent, the barrier of the RE is lowered. However, not enough, to become the lowest energy pathway as one would expect from chemical intuition. Using explicit solvent molecules, we found that their interaction with the hypervalent bond is too complex to be adequately described by a pure electrostatic solvent model.

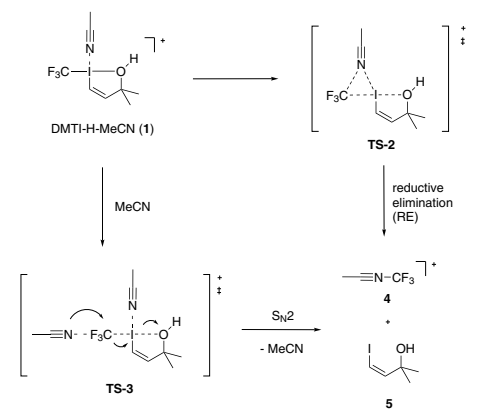

[1] K. Niedermann, N. Fruh, E. Vinogradova, M.S. Wiehn, A. Moreno, A. Togni, Angew. Chem. Int. Ed. 2011, 50, 1059-1063. 
Computational Chemistry

\section{A key weak point of the prion protein}

\author{
$\underline{\text { Julian Garrec }}^{1}$, co-authors
}

${ }^{1}$ École Polytechnique Fédérale de Lausanne, Laboratory of Computational Chemistry and Biochemistry, Institute of Chemical Sciences and Engineering, CH-1015 Lausanne, Switzerland

The characteristic misfolding of the prion protein (PrP) observed in transmissible spongiform encephalopathies (TSE) is favored by acidic $\mathrm{pH}$ in vitro, which represent relevant conditions insofar as endosome organelles have been proposed as possible location for PrP aggregation. Recent experiments have shown that the protonation of $\mathrm{H} 187$ in the prion protein $(\operatorname{PrP})$ at endosomal $\mathrm{pH}$ induces a conformational transition of the protein that could be linked to characteristic misfolding of prion diseases. Using molecular dynamics simulations, we have explored the mechanism of the early stages of this destabilization process. Our results suggest a model in which the protonation of H187 induces an electrostatic repulsion with the nearby guanidinium group of R136. The system responds to this perturbation by pushing either H187 or R136 away from their native cavity. This triggers a cascade of conformational transitions in different regions of the protein, some of which are located far from H187. The finding that the H187 interaction with R136 is a weak point of mammalian PrP is supported by the absence of the $\{H 187, \mathrm{R} 136\}$ residue pair in non-mammalian species that are known to be resistant to TSE. The imidazole ring of $\mathrm{H} 187$ and the guanidinium group of R136 form a solventaccessible site that represent an alternative or complementary target for drug design.
Computational Chemistry

Non-bonded Interactions in Solution:

Interplay between Theory and Experiment

\section{Stephan N. Steinmann and Clemence Corminboeuf}

Laboratory for Computational Molecular Design, Institute of Chemical Sciences and Engineering, Ecole Polytechnique Fédérale de Lausanne, 1015 Lausanne, Switzerland

Non-bonded interactions are responsible for many structural and energetical phenomena, which are difficult to assess experimentally. The present computational approach aims at providing a comprehensive picture of these processes that complements experimental data. We here specifically decipher the origin of the selectivity of chemosensors and provide details regarding the position of individual molecules in self-assembled soft matter. Achieving good agreement with experiment relies on considering both explicit solvent effects and intermolecular flexibility.

We estimate the selectivity of molecular receptors for caffeine $e^{1}$ accounting for explicit solvent and entropy effects by combining the quantum mechanical treatment of the intermolecular complex with molecular mechanics simulations in aqueous solution. Similarly, key structural parameters of supramolecular assemblies are extracted from classical trajectories. Those structures, which combine bioinspired building blocks and $\pi$-conjugated moieties are promising precursors to organic electronics. Information on relative arrangements between adjacent $\pi$-functional units helps identifying the most promising candidate structures.
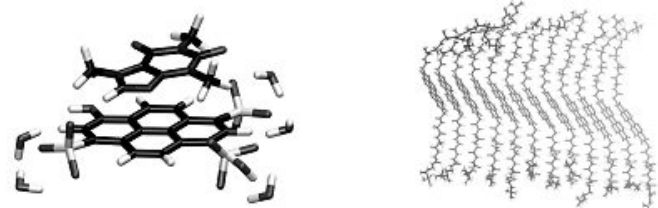

[1] S. Rochat, S.N. Steinmann, C. Corminboeuf, K. Severin, Chem. Commun. 2011, 47, 10584.

Computational Chemistry

Investigating the nature of the charge carriers in conducting organic polymers using DMRG

\section{$\underline{\text { L. Bomble, J. Gonthier, R. Petraglia, C. Corminboeuf }}{ }^{1}$}

${ }^{1}$ Laboratory for Computational Molecular Design, EPFL, Switzerland

The Density Matrix Renormalization Group (DMRG) ansatz has been developed in the 1990 's by the solid state physics community to enable the study of large-scale systems with strong electronic interactions. DMRG has recently emerged as a solution to challenging quantum chemistry problems [1]. The DMRG protocol [2] defines an effective Hamiltonian that focuses on the most important correlation interactions and iteratively approaches the full CI (Configuration Interaction) solution. The approach produces very accurate results for large systems (up to $100 \pi$ electrons in 100 orbitals) [3-4] but is still at a development stage. Our goal is to implement and use the DMRG protocol to investigate the polaron-bipolaron nature of conductive organic polymers with increasing chain size [5]. These molecules, which are key players in the field of organic electronics, are very challenging for standard electronic structure methods. Long polymer chains are indeed characterized by strong multireference character but are too large to be treated using high-level multireference ab initio methodologies (up to 20 monomers, i.e. 120 pi electrons). Alternatively, Kohn Sham Density Functional Theory (DFT) has been extensively used but there exists no accurate reference to validate the diverse performance of standard and modern functionals. Our aim is to use DMRG to benchmark the accuracy of density functional computations and determine the nature of the charge carriers in conductive organic polymers.

[1] S. R. White and R. L. Martin J. Chem. Phys, 110, 4127. [2] G. K.-L. Chan and M. Head-Gordon J. Chem. Phys, 116, 4462. [3] K. H. Marti , I. M. Ondik, G. Moritz and M. Reiher J. Chem. Phys, 128, 014105. [4] J. Hachmann, W. Cardoen, and G. K.-L. Chan J. Chem. Phys, 125,14410. [5] T. Nishinaga, M. Tateno, M. Fujii, W. Fujita, M. Takase, M. IyodaOrg. Let., 12,5374.
[1] M. Elstner et al., Phys. Rev. B 1998, 58, 11.
[2] Stephan N. Steinmann and Clemence Corminboeuf, J. Chem. Teory Comput. 2011, 7, 3567.

[3] K. T. Tang and J. P. Tonnies, J. Chem. Phys. 1984, 80, 3726. 
Computational Chemistry

Unraveling the Mechanism of the Unusual Topochemical

\section{Dimerization of Bromodiacetylene}

Tanya K. Todorova and Clemence Corminboeuf

Laboratory for Computational Molecular Design, Ecole Polytechnique Fédérale de Lausanne, CH-1015 Lausanne, Switzerland

Topochemical reactions of organic molecules in the crystalline state often proceed with unrivalled selectivity and sometimes allow for reactions not conceivable in solution. ${ }^{1}$ Upon exposure to light, bromodiacetylene undergoes an unusual dimerization reaction, which proceeds under drastic displacements and rearrangements of the reacting molecular fragments. ${ }^{2}$ An investigation of the underlying mechanism by means of DFT computations suggests that the rate-determining initial step is a $[2+1]$ photocycloaddition that appears to be predetermined by the nature of short contacts between pairs of molecules in the crystalline state. ${ }^{2}$ Insight into the electronic structure of all the stationary is provided by the localized orbital locator (LOL), proven to be a very useful tool in assigning chemical bonding patterns in complex systems. ${ }^{3}$

[1] L. R. MacGillivray, G. S. Papaefstathiou, T. Friscic, T. D. Hamilton, D.K. Bucar, Q. Chu, D. B. Varshney, I. G. Georgiev, Acc. Chem. Res. 2008 , $41,280$.

[2] T. N.Hoheisel, S. Schrettl, R. Marty, T. K. Todorova, C. Corminboeuf, R. Scopelliti, W. B. Schweizer, H. Frauenrath, Natur. Chem. (submitted). [3] S. N. Steinmann, Y. Mo, C. Corminboeuf, Phys. Chem. Chem. Phys. 2011, 13, 20584
Computational Chemistry

Mutations and Deletions to Investigate the Dynamics of Group II Introns by smFRET

Fiorini, E.; Cardo, L.; Kowerko, D. and Sigel, R.K.O.

University of Zurich, Institute of Inorganic Chemistry, Winterthurerstrasse 190,8057, Zurich (Switzerland), +41 446354652, erica.fiorini@uzh.ch

Group II introns belong to the class of self-splicing ribozymes, are mobile genetic elements and are found within the genes of bacteria and lower eukaryotes [1]. We designed a derivative of $S$. Cerevisiae ai5 $\gamma$ group II intron ( $\sim 900 \mathrm{nts}$ ), named D135-L14, which is labeled with the Cy3-Cy5 fluorophores. Construct allows for a detailed characterisation of the folding pathways by smFRET using TIRF microscopy since it is able to preserve the dynamics and the catalytic activity of the parent ribozyme are preserved [2]. The group II intron ribozyme folds sequentially in three steps from the unfolded to the native state with distinguishable structural conformations, whose related rate constants depend on concentration and the type of metal ions in the system $[2,3]$. We now introduced point mutations and/or domain deletions in those positions responsible for inter-domain docking. Subsequent smFRET analysis of these derivatives allowed us to assign the specific structures to each FRET state of the folding process.

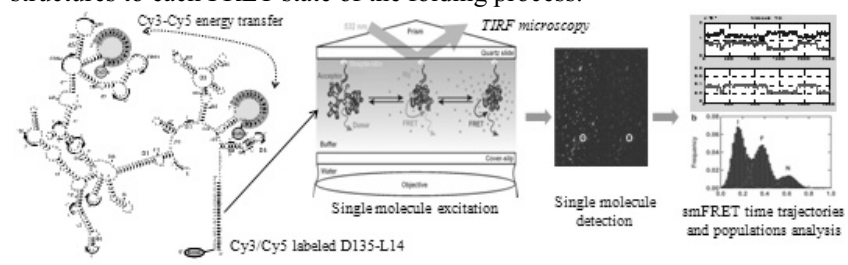

References

[1] A.M. Pyle, Crit. Rev. Biochem. Mol. Biol., 2010, 45, 215

[2] M. Steiner, K.S. Karunatilaka, R.K.O. Sigel, D. Rueda, PNAS, 2008, 105 , 13853.

[3] L. Cardo, K. Karunatilaka, D. Rueda and R.K.O. Sigel (2012) Methods Mol. Bio.l, 2012, 848, 227.
Computational Chemistry

Insight of trHbN: quantitative analysis of the network and gate-trapping for $\mathrm{O2}$ uptake.

$\underline{\text { Pierre-André Cazade }}^{1}$, Florent Hedin ${ }^{1}$, Markus Meuwly ${ }^{1 *}$

${ }^{1}$ Department of Chemistry, University of Basel, Klingelbergstrasse 80, $\mathrm{CH}-4056$ Basel

Truncated hemoglobin (trHbN) is a protein involved in NO dioxygenation $[1,2]$ by converting nitric oxide to harmless nitrates $\left(\mathrm{Fe}(\mathrm{II}) \mathrm{O}_{2}+\mathrm{NO} \rightarrow\right.$ $\left.\mathrm{Fe}^{+}(\mathrm{III})+\mathrm{NO}_{3}^{-}\right)$. The tunnel system of trHbN plays an important role in determining and controlling ligand entrance, migration, and rebinding.[2] Due to a greater affinity of $\mathrm{Fe}$ (II) for $\mathrm{NO}$ than for $\mathrm{O}_{2}$, the first step of dioxygenation is expected to be the substitution of NO ligated to the heme group by free $\mathrm{O}_{2}\left(\mathrm{Fe}(\mathrm{II}) \mathrm{NO}+\mathrm{O}_{2} \rightarrow \mathrm{Fe}(I I) \mathrm{O}_{2}+\mathrm{NO}\right)$. $\mathrm{O}_{2}$ dynamics and migration in the protein network has already been analyzed [3], providing the ligand docking sites and their connectivity together with the free energy barriers between them. The network can be further analyzed by means of a Transition Network Analysis (TNA) which provides a comprehensive picture of the ligand migration dynamics and kinetics in the network from several independent trajectories [4]. It proves typically the time-resolved connectivity network in the protein, the half-lives of the docking sites, the transition timescales, and the extent of population transfer among different docking sites. Furthermore, a close attention at the coupling between ligand and protein dynamics reveals a gate-trapping mechanism for the ligand uptake. The protein conformation changes are studied by means of MD and MC simulations.

[1] Hausladen, A., Gow, A., Stamler, J. S., Proc. Natl. Acad. Sci. U.S.A. 2001, 98, 10108-10112.

[2] Mishra, S., Meuwly, M., J. Am. Chem. Soc., 2010, 132, 2968.

[3] Cazade, P.-A., Meuwly, M., Oxygen Migration Pathways in Truncated Hemoglobin, submitted, 2012.

[4] Mishra, S., Meuwly, M., Biophysical J., 2010, 99, 3969.
Computational Chemistry

Sampling Rare Events with Spatial Averaging: Implementation and Application to Rare Gas Clusters and Biomolecules

$\underline{\text { Florent Hédin }}^{1}$, Nuria Plattner ${ }^{2}$, Markus Meuwly $^{3 *}$

$12{ }^{3}$ Department of Chemistry, University of Basel, Klingelbergstrasse 80, CH-4056 Basel

Spatial averaging [1] is an efficient Monte Carlo method which can be applied to problems where important regions (e.g. minima) of the energy landscape may be difficult to sample with a standard random walk method, such as Metropolis sampling. At the heart of the method is the realisation that from the equilibrium density a related, modified probability density can be constructed through a suitable transformation. This new density is more highly connected which increases the chances for transitions between neighbouring states which in turn speeds up the sampling. In order to transform the equilibrium density, Gaussian distributions with variable widths are used.

First successful investigations included the diffusion of small molecules in condensed phase environments [2] and localisation of lowest energy structures of Lennard-Jones clusters [3]. A more general implementation in CHARMM allowed us to study the conformational space of small biomolecules.

[1] JD Doll, JE Gubernatis, N Plattner, M Meuwly, P Dupuis, and H Wang, JOURNAL OF CHEMICAL PHYSICS 2009, 10, 131.

[2] N Plattner, JD Doll, and M Meuwly, JOURNAL OF CHEMICAL PHYSICS 2010, 4, 133.

[3] F. Hédin, Master Thesis, University of Basel 2011 
Computational Chemistry

DFT study of the Jahn-Teller and Pseudo-Jahn-Teller effects in the excited states of $\mathrm{CO}_{3}$

\section{$\underline{\text { Ramanantoanina Harry }}^{1}$, Daul Claude}

${ }^{1}$ University of Fribourg, Chemin du Musée 9, 1700 Fribourg

The electronic structure and the geometry of $\mathrm{CO}_{3}$ have been the subject of tremendous interest [1], since the experimental evidence of the conformation of this molecule in $D_{3 h}$ [2] point group was reported, in addition to the well-known $C_{2 v}$ symmetry [3]. The $\mathrm{CO}_{3}$ molecule is involved as intermediate reagent in the atmospheres of Earth and Mars for quenching electronically excited oxygen atoms and in contributing to the ${ }^{18} \mathrm{O}$ isotope enrichment of stratospheric carbon dioxide [2]. Herein, we report a discussion about the ground state configuration and the geometry of the $\mathrm{CO}_{3}$ molecule: $C_{2 v}$ versus $D_{3 h}$ by means of the Density Functional Theory (DFT). The presence of the $C_{2 v}$ conformation, which is the global minimum, lower in energy about $500 \mathrm{~cm}^{-1}$ than the $D_{3 h}$ local minimum is reported, and the corresponding Adiabatical Potential Energy Surface of the descent in symmetry is analyzed. The combination of the Jahn-Teller Effect and the Pseudo-JahnTeller Effect, which are involved in the twofold degenerate ${ }^{1} E^{\prime}$ excited states of the conformation in the $D_{3 h}$ point group are mainly responsible of the distortion. Our Density Functional Theory analysis of this small molecule with a complicated electronic structure is efficient and in agreement with the previous work reported by Liu, Bersuker et al., [4] based on a very high level quantum chemistry ab initio method.

[1] (a) Mebel, A.M.; Hayashi, M.; Kislov, V.V.; Lin, S.H.; J. Phys. Chem A 2004, 108, 7983; (b) Benett, C.J.; Jamieson, C.; Mebel, A.M.; Kaiser, R.I.; Phys. Chem. Chem. Phys., 2004, 6, 735.

[2] Jamieson, C.A.; Mebel, A.M.; Kaiser, R.I.; ChemPhysChem. 2006, 7, 2508 .

[3] (a) Moll, N.G.; Clutter, D.R.; Thompson, W.E.; J. Chem. Phys., 1966 45, 4469; (b) Jacox, M.E.; Milligan, D.E.; J. Chem. Phys., 1971, 54, 919.

[4] Liu, Y.; Bersuker, I.B.; Zou, W.; Boggs, J.E.; J. Chem. Theory Comput., 2009, 5, 2679.
Computational Chemistry

200

Theoretical Investigation to Study Organic Photo sensitizers dyes

\section{$\underline{\text { Sofiane Moussi }}{ }^{1,2}$, Claud Daul $^{2}$, Ourida Ouamerali ${ }^{1}$}

University of Fribourg, Chemin du musée 9, 1700 Fribourg / ${ }^{2}$ University of Science and Technology Houari Boumedienne / El alia Pobox 32 Bebezzouar, 16111 Algiers, Algeria

A novel class of organic photo sensitizers is sought ${ }^{[1,2]}$. This organic dyes Pyrenoimidazole- $\pi$ bridge-Cyanoacrylic acid show charge migration from the donor to the acceptor through the $\pi$-bridge (Donor- $\pi$ bridgeAcceptor). The intensity and the position of the charge transfer transition can be tuned by simple structural modification (variation of donor strength and nature of the conjugation path way ${ }^{[3]}$.

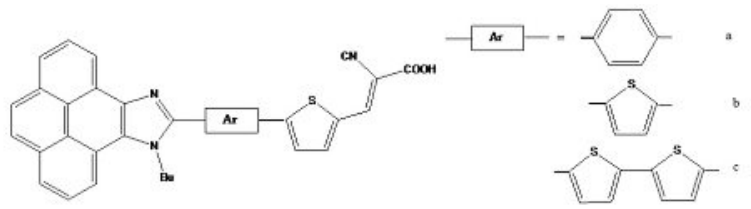

In this study we use both TD DFT and Multi determinantal DFT calculation to tune the $\pi \rightarrow \pi^{*}$ energy gap in order to cover the whole spectral range of sun light.

[1] Chenyi Yi, Carmen Blum, Mario Lehmann, Stephan Keller, Shi-Xia Liu, Gabriela Frei, Antonia Neels, Jurg Hauser, Stefan Schurch, Sil vio Decurtins, J.Org.Chem. 2010, 75, 3350.

[2] Dhirendra Kumar, K. R. Justin Thomas, Chuan Pei Lee, Kuo-Chuan Ho, journal Org. Lett, 2011, Vol 13, No. 10, 2622.

[3] Mishra,A.;Fisher,M. K. R.;Bauerel,P. Angew. Chem., Int. Ed, 2009, 48,2474

Computational Chemistry

Computational Chemistry

Correlation between the vibrational properties of borohydride compounds and the local environment of $\mathrm{BH}_{4}{ }^{-}$: a theoretical analysis

\author{
Vincenza D’Anna, Latévi Max Lawson Daku, Hans Hagemann \\ Université de Genève 30, Quai Ernest-Ansermet \\ CH-1211 Genève 4
}

Due to their high gravimetric and volumetric hydrogen content, inorganic borohydrides are considered as potential solid hydrogen storage material for mobile applications.

For this reason it is necessary to achieve an in-depth understanding of the relationship between their bonding properties and their structures.

From this point of view, vibrational spectroscopy can be considered a valuable approach because the vibrational spectra of $\mathrm{BH}_{4}^{-}$are sensitive to its local environment.

Here we try to relate the vibrational properties of $\mathrm{BH}_{4}{ }^{-}$to its local symmetry, from the analysis of a comprehensive database of experimental infrared and Raman spectra based on the FG method [1] and on molecular and periodical calculations performed within Density Functional Theory [2,3].

\section{[1] E. B. Wilson, J. Chem. Phys. 1939, 7, 1047}

[2] P. Hohenberg and W. Kohn, Phys. Rev. 1964, 136, B864

[3] W. Khon and L. J. Sham, Phys. Rev. 1965, 140, A1133
Visualization and Rapid Search of Chemical Space with Scalar Fingerprints

$\underline{\text { Julian Schwartz }}^{1}$ and Jean-Louis Reymond ${ }^{1}$

${ }^{1}$ Department of Chemistry and Biochemistry, University of Berne

Binary substructure fingerprints in combination with the Tanimoto coefficient as distance metric are commonly used to perform in silico similarity searches in virtual chemical databases.

We have recently shown that scalar fingerprints of molecules, like the 42 Molecular Quantum Numbers (MQN ${ }^{[3]}$ descriptor set, can be used for rapid Nearest-Neighbor searches in large databases. ${ }^{[1]}$ Moreover, the MQN set is able to retrieve annotated bioactive compounds from PubChem with significant enrichment factors. ${ }^{[2]}$

Herein, we demonstrate the usefulness of scalar fingerprints, in particular, of a simple 34 dimensional scalar descriptor set that can be readily derived from SMILES, and allows for rapid similarity searches and significant enrichments of bioactive compounds from large chemical databases. As such, the chemical universe database GDB-13 ${ }^{[1]}$, (975 million molecules) can be searched within seconds.

Additionally, an n-dimensional scalar molecular descriptor set, as opposed to binary molecular descriptor sets (usually 1024 dimensional), can be interpreted as an n-dimensional Chemical Space where molecules with similar descriptors, and thus similar structures and presumably similar bioactivities, cluster in the same region of the chemical space. Due to the small number of dimensions, the resulting property space is chemically meaningful and can be visually inspected, after application of principle component analysis, as a two-dimensional projection of the 34-dimensional space.

[1] L. C. Blum et. al., J. Comput. Aided Mol. Des. 2011, 25, 637.

[2] R. v. Deursen et. al., J. Comput. Aided Mol. Des. 2011, 25, 649.

[3] K. T. Nguyen et. al., ChemMedChem 2009, 4, 1803. 
Computational Chemistry

203

Effects of the finite length of the pump laser pulse in nonadiabatic quantum dynamics simulations of ultrafast time-resolved spectroscopy

Aurélien Patoz, Marius Wehrle, and Jiri Vanicek

Ecole Polytechnique Fédérale de Lausanne

We have implemented a general split-operator algorithm for exact nonadiabatic quantum dynamics of a molecule interacting with a time-dependent electromagnetic field. This algorithm enables evaluation of the accuracy of the assumption of ultrashort pulse length as well as of other approximations frequently used in theoretical treatments of such systems. We compare the usual approximate treatments with the exact solution for the ultrafast nonadiabatic dynamics of a four-dimensional model of pyrazine.
Computational Chemistry

Understanding the nucleation of the microRNA-mRNA pairing by using CLIP-Seq and RNA folding data

\section{Ray Marín ${ }^{1}$, Jiří Vaníček ${ }^{1}$}

${ }^{1}$ Laboratory of Theoretical Physical Chemistry, Institut des Sciences et Ingnierie Chimiques, cole Polytechnique Fdrale de Lausanne, $\mathrm{CH}-1015$

Lausanne, Switzerland

To find out whether the AGO-miRNA complex is more sensitive to the accessibility of a particular region inside the seed match, we analyze in detail the accessibility of a wide set of miRNA binding sites validated by PAR-CLIP experiments [1]. Our analysis reveals that nucleotides at the 3'-end of bound seed matches are significantly more accessible than nucleotides at the 5'-end, and also more accessible than nucleotides at any positions in the unbound seed matches. We show that the accessibility of a single nucleotide at the 3 '-end is more effective than the accessibility of several nucleotides at the 5 '-end in discriminating between functional and non-functional sites. Analysis of mRNA and protein fold changes induced by miRNA over-expression, demonstrated that genes with accessible nucleation regions at the 3'-end are more strongly downregulated than genes whose accessible nucleation regions are located elsewhere within the seed match. The pronounced sensitivity of the AGO-miRNA complex to the accessibility of the 3'-end of the seed match suggests that, in most cases, nucleation occurs in this region. It is shown that our conclusions are consistent with previous experimental studies [2-4].

[1] M. Hafner, M. Landthaler, L. Burger, M. Khorshid, J. Hausser, P. Berninge A. Rothballer, M. Ascano Jr, et al., Cell 2010, 141, 129.

[2] Y. Wang, G. Sheng, S. Juranek, T. Tuschl, and D.J. Patel, Nature 2010, 456, 209.

[3] S.L. Ameres, J. Martinez, and R. Schroeder, Cell 2007, 130, 101.

[4] S.W. Chi, G.J. Hannon, and R.B. Darnell, Nat. Struct. Mol. Biol. 2012, 19,321 .
Computational Chemistry

Ultrafast time-resolved electronic spectra with on-the-fly ab initio semiclassical methods for many-dimensional systems

\section{Wehrle, J. Vaníček*}

Laboratory of Theoretical Physical Chemistry, Institut des Sciences et Ingénierie Chimiques, École Polytechnique Fédérale de Lausanne, CH-1015

$$
\text { Lausanne, Switzerland }
$$

Semiclassical methods allow to capture quantum effects approximately but they are usually difficult to apply to high-dimensional systems because of main two reasons: First, the number of trajectories required often scales exponentially with the number of degrees of freedom. Second, semiclassical algorithms typically require the Hessian of the potential in addition to the force, which makes the calculation more expensive than classical calculations. It has been showed that the required number of trajectories is independent of dimensions for the dephasing representation of quantum fidelity $[1,2]$. The capability of the dephasing representation for "on-the-fly" quantum simulations of large systems is demonstrated by calculating the timeresolved stimulated emission spectrum of the 54-dimensional azulene molecule.

[1] J. Vaníček, Phys. Rev. E 2006, 73, 046204.

[2] C. Mollica, J. Vaníček, Phys. Rev. Lett. 2011, 107, 014101.

*jiri.vanicek@epfl.ch

Accelerating the calculation of time-resolved electronic spectra of many-dimensional systems with the cellular dephasing representation

\section{Miroslav Šulc, Jiří Vaníček*}

Institut des Sciences et Ingénierie Chimiques,

Ecole Polytechnique Fédérale de Lausanne, CH-1015, Switzerland

Dephasing representation (DR) of fidelity [1], also known as the phase averaging method, can be considered as a special case of Miller's linearised semiclassical initial value representation and belongs among the most efficient approximate semiclassical approaches for the calculation of ultrafast time-resolved electronic spectra.

It has been shown [2] that the number of trajectories required for convergence of the DR is independent of the dimensionality. Here we propose [3] a further accelerated version of the dephasing representation in the spirit of Heller's cellular dynamics. The basic idea of the "cellular dephasing representation" is to decompose the Wigner transform of the initial state into a phase space Gaussian basis and then evaluate the contribution of each Gaussian to the relevant correlation function approximately analytically, using numerically acquired information only along the trajectory of the Gaussian's centre.

The proposed method turns out to be sufficiently accurate whenever the interaction with the environment diminishes the importance of recurrences in the correlation functions of interest. Numerical tests on a collinear NCO molecule and on a 48-dimensional azulene model indicate that even 1-trajectory results are in good agreement with the fully converged DR ( $10^{4}$ trajectories).

[1] J. Vaníček, Phys. Rev. E 2006, 73, 046204.

[2] C. Mollica, J. Vaníček, Phys. Rev. Lett. 2011, 107, 214101.

[3] M. Šulc, J. Vaníček, Mol. Phys. 2012, published online DOI: $10.1080 / 00268976.2012 .668971$.

*E-mail: jiri.vanicek@epfl.ch 
Computational Chemistry

Measuring nonadiabaticity and importance of spin orbit couplings in the quantum molecular dynamics using the quantum fidelity and its efficient semiclassical approximation

Tomáš Zimmermann, Jiří Vaníček ${ }^{\star}$

Laboratory of Theoretical Physical Chemistry, Institut des Sciences et Ingénierie Chimiques, Ecole Polytechnique Fédérale de Lausanne, CH-1015, Switzerland

We propose to measure nonadiabaticity of molecular quantum dynamics rigorously by the quantum fidelity between the Born-Oppenheimer quantum dynamics and the full nonadiabatic quantum dynamics. In addition to nonadiabaticity, the quantum fidelity may be used to evaluate the importance of the spin orbit couplings and other coupling terms. The quantum fidelity is estimated efficiently with the multiple-surface dephasing representation (MSDR) [1], which is the generalization of the dephasing representation (DR) [2] of quantum fidelity to several potential energy surfaces (PESs). In contrast to quantum dynamics the cost of the MSDR does not scale exponentially with the number of degrees of freedom. Being a semiclassical method, it still approximately includes quantum effects on the motion of nuclei through in terference without the need for the Hessian of PESs. Two mixed quantumclassical dynamics methods are currently used to propagate the nuclei: the locally mean field method (LMFD), which turns out to be nothing else than the Ehrenfest dynamics of an ensemble of trajectories and the fewest switches surface hopping dynamics (FSSH). The method is compared to the numerically exact quantum results using several model problems. Subsequently, it is applied on-the-fly to evaluate the nonadiabaticity and the importance of spin orbit couplings in the dynamics on the second excited state of azulene and $\mathrm{CH}_{2} \mathrm{NH}_{2}^{+}$and in the collision of $\mathrm{F}+\mathrm{H}_{2}$.

[1] T. Zimmermann and J. Vaníček, J. Chem. Phys. 2012, 136, 094196.

[2] J. Vaníček, Phys. Rev. E 2004, 70, 055201; J. Vaníček, Phys. Rev. E 2006, 73, 046204 .

Evaluation of Parity Violating Energy Differences for Cyanoaziridine, Cyano- and Fluorooxiranes with Highly Correlated Wavefunctions.

\section{Luboš Horný and Martin Quack}

Physical Chemistry, ETH Hönggerberg, CH-8093 Zürich, Switzerland

Possible experiments on molecular parity violation and its possible implications for the origin of molecular chirality and biomolecular homochirality are of fundamental interest ${ }^{1,2}$. It has been established ${ }^{3}$ how to include the electron correlation effects in computation of molecular parityviolation potentials $\mathrm{E}_{\mathrm{pv}}$ using nonrelativistic highly correlated coupled cluster wavefunctions within a linear response framework ${ }^{4}$. This complements previous developments in the precise theoretical description of molecular-parity violation ${ }^{5,6}$. Recently, high resolution spectroscopic analysis of chiral compounds became feasible ${ }^{1,2,7}$. We report structure and energetics for some of such species - cis-/trans-cyanoaziridine, cyano- and fluorooxirane, and compare them to previous results ${ }^{2,8}$. Using $\mathrm{CC} 2$ and CCSD wavefunctions, we find (R)-configurations of cyanoaziridine, and fluorooxirane, and (S)-configuration of cyanooxirane to be energetically more stable than their counterparts.

[1] M. Quack, "Fundamental Symmetries and Symmetry Violations from High Resolution Spectroscopy" In Handbook of High Resolution Spectroscopy, M. Quack and F. Merkt eds. 2011, 659.

[2] M. Quack, J. Stohner, M. Willeke, Ann. Rev. Phys. Chem. 2008, 59, 741.

[3] L. Horný and M. Quack, Faraday Discussions 2011, 150, 152.

[4] T. Helgaker, S. Coriani, P. Jørgensen, K. Kristensen, J. Olsen, K. Ruud,

Chem. Rev. 2012, 112

[5] A. Bakasov, T. K. Ha, M. Quack, J. Chem. Phys. 1998, 109, 7263.

[6] R. Berger and M. Quack, J. Chem. Phys. 2000, 112, 3148.

[7] Albert S. and M. Quack, ChemPhysChem 2007, 8, 1271.

[8] R. Berger, M. Quack, G. S. Tschumper, Helv. Chim. Acta, 2000, 83, 1919.

[9] R. Berger, M. Quack, J. Stohner, Angew. Chem. Intl. Ed. 2001, 40, 1667.
Computational Chemistry

Study of Th(IV) hydration structure by theoretical methods

Beuchat César $^{1}$, Spezia Riccardo ${ }^{2}$ and Gagliardi Laura ${ }^{3}$

${ }^{1}$ University of Geneva, Department of Physical Chemistry, Quai Ernest Ansermet 30, CH-1211 Geneva,

${ }^{2}$ CNRS, LAMBE UMR 8587, Université d'Evry Val d'Essonne, Evry, France, 91025, France

${ }^{3}$ University of Minnesota, Department of Chemistry and Supercomputing Institute, 207 Pleasant St. SE,

The understanding of hydration properties of ions is the first step to study their reactivity in water. In particular, properties like ion-water distances and coordination number of solvation shells are at the centre of both theoretical $^{1,2}$ and experimental studies.

Thorium is of particular interest because it can be used as a combustible for nuclear power plants. This could in principle lead to nuclear energy based on a more abundant combustible and with less radioactive wastes. Thorium(IV) hydration has been studied experimentally and theoretically, but many questions are still unanswered. In particular, while it is well established that the Th-water first shell distance is $2.45-2.46 \AA$, the coordination number is not yet uniquely defined. EXAFS and HEXS experiments have provided values in the 8-12 range.

Given the interest in the topic, we have undertaken a computational study aimed at a deeper understanding of the Th(IV) hydration structure and the differences between $\mathrm{ThCl}_{4}$ and $\mathrm{ThBr}_{4}$ salts at different concentrations. Several theoretical methods were employed: density functional theory, classical molecular dynamics based on both semi-empirical polarisable potentials and $\mathrm{ab}$ initio derived polarisable potentials. The results of the computations were combined with EXAFS experimental data.

[1] C.Beuchat, D.Hagberg, R.Spezia, L.Gagliardi, J. Phys. Chem. B. 2010, 114 (47), 15590.

[2] M.Duvail, P.Vitorge and R.Spezia, J. Chem. Phys. 2009, 130, 104501.

Computational Chemistry

Predicting Molecular Properties with Machine Learning

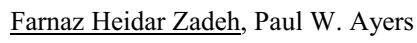

McMaster University, Department of Chemistry and Chemical Biology, 1280 Main Street West, Hamilton, Ontario, Canada

Determining the properties of molecules has always been one of the major tasks of chemical research. When one is searching for an existing molecule with a desired property, or trying to design a new molecule with a desired property, one often needs to consider thousands of possible compounds. This makes the experimental and computational material property determination of innumerable possibilities costly and impractical in most cases. On the other hand, there are many properties that are not directly measurable experimentally or computable theoretically. For example, one can look at the toxicity of molecules: it would be unethical to test these molecules experimentally, and it is computationally intractable to simulate the ingestion, digestion, and biochemical response of a molecule.

However, by exploring the machine learning methods, we can efficiently, and with controlled errors, estimate the properties of a chemical substance by leveraging the results from previous experiments or accurate calculations. The key insight is that similar molecules tend to have similar properties. So if the properties of a diverse "reference set" of molecules is known, then one can predict the properties of unknown molecules (the "test set") by determining the relationship between the unknown molecules and the reference molecules [1]. The approach we are developing for molecular similarity is based on kriging, a powerful estimation tool originally used in geostatistics [2]. In this presentation, this method is applied to predict various molecular and material properties.

[1] Burger, S. K.; Liu, S. B.; Ayers, P. W. J. Phys. Chem. A, 2011, 115, 1293.

[2] Wackernagel, H., Multivariate Geostatistics: An introduction with applications. Springer-Verlag: New York, 2003. 